Sánchez Martín J. M., Rengifo Gallego J. I., Sánchez Rivero M. (2017): "Caracterización espacial del turismo en Extremadura mediante análisis de agrupamiento (Grouping Analysis). Un ensayo técnico”, GeoFocus (Artículos), $n^{\circ} 19$, p. 207-235. ISSN: 1578-5157. http://dx.doi.org/10.21138/GF.490

\title{
CARACTERIZACIÓN ESPACIAL DEL TURISMO EN EXTREMADURA MEDIANTE ANÁLISIS DE AGRUPAMIENTO (GROUPING ANALYSIS). UN ENSAYO TÉCNICO
}

\author{
JOSÉ MANUEL SÁNCHEZ MARTÍN ${ }^{1}$, JUAN IGNACIO RENGIFO GALLEGO², \\ MARCELINO SÁNCHEZ RIVERO ${ }^{3}$ \\ ${ }^{1}$ Facultad de Empresa, Finanzas y Turismo \\ Avda. de la Universidad S/N 10003, Cáceres, España \\ jmsanche@unex.es \\ ${ }^{2}$ Facultad de Filosofía y Letras \\ Campus Universitario, S/N 10003, Cáceres, España \\ irengi@unex.es \\ ${ }^{3}$ Facultad de Ciencias Económicas y Empresariales \\ Avda. de Elvas, 6071, Badajoz, España \\ sanriver@unex.es
}

\section{RESUMEN}

Este artículo aborda un ensayo técnico de caracterización del espacio turístico de Extremadura a partir de la aplicación de Análisis de Grupos o de Agrupamiento (Grouping Analysis), técnica vinculada a la geoestadística. Se consideran variables relativas a los atractivos y la oferta de alojamientos y restauración y, además, se recurre al criterio espacial. Con ello, se trata de ir más allá de los análisis exclusivamente cuantitativos, al incorporar el espacio próximo como factor destacado a la hora de establecer grupos homogéneos. Se realizan numerosos ensayos, utilizando variables muy diversas y diferentes criterios de restricción espacial, desde la omisión del mismo, hasta la consideración de tres vecinos como criterio de distancia. Se pone de manifiesto que los resultados que ofrece la utilización de éste último criterio son sensiblemente mejores que los obtenidos con la omisión del aspecto espacial. Como consecuencia de su aplicación, se obtienen 14 áreas turísticas bien diferenciadas. turístico.

Palabras clave: Análisis de agrupamiento, restricción espacial, Extremadura, territorio 
Sánchez Martín J. M., Rengifo Gallego J. I., Sánchez Rivero M. (2017): “Caracterización espacial del turismo en Extremadura mediante análisis de agrupamiento (Grouping Analysis). Un ensayo técnico”, GeoFocus (Artículos), $n^{\circ} 19$, p. 207-235. ISSN: 1578-5157. http://dx.doi.org/10.21138/GF.490

\title{
SPATIAL CHARACTERIZATION OF THE TOURISM IN EXTREMADURA (SPAIN) USING GROUPING ANALYSIS. A TECHNICAL TEST
}

\begin{abstract}
This paper shows a technical test on the characterization of the tourist space of Extremadura by applying Grouping Analysis, a technique linked to geostatistics. Variables related to tourist attractions and accommodation and catering supply are considered, as well as spatial criteria. Thus, it tries to go beyond a mere quantitative analysis by incorporating the neighboring space as a key factor in establishing homogeneous groups. Numerous tests are performed, using quite diverse variables and different spatial restriction criteria, since its omission, to the consideration of three neighbors as a criterion of distance. It is shown that the results obtained after the inclusion of the latter criterion are significantly better than those obtained after the omission of the spatial feature. As a result of this application, 14 well-differentiated tourist areas are obtained.
\end{abstract}

Keywords: Grouping Analysis, spatial restriction, Extremadura, tourist territory.

\section{Introducción}

El análisis del sector turístico posee una fuerte componente espacial, pues buena parte de las variables turísticas está condicionada por los valores que toman en lugares próximos al destino. Esta circunstancia hace posible utilizar variadas técnicas estadísticas para su análisis (Sánchez, 2008). Éstas permiten realizar análisis pormenorizados del territorio, a la vez que cuantitativos, ya que consideran las interrelaciones entre los casos analizados en un contexto variable, determinado por el espacio próximo como un elemento destacable. Aportan la concepción territorial y sus interconexiones como un valor añadido al tradicional análisis cuantitativo o cualitativo en que se basa la estadística. Dicha peculiaridad permite un estudio integrado e inserto en el contexto espacial inmediato, circunstancia que resulta clave para entender la generación de áreas turísticas homogéneas, pero también para generar productos turísticos análogos o complementarios, marcas territoriales de calidad, etc.

En líneas generales, el conjunto de técnicas geoestadísticas permite explicar buena parte de los elementos que definen el turismo y su distribución territorial, pues ésta no es uniforme en el espacio analizado, aunque tampoco presenta síntomas de aleatoriedad. Un análisis sosegado de la distribución del hecho turístico en un lugar dado permite extraer algunas pautas, a veces difusas, pero visibles cuando se analizan desde la óptica geográfica. Por ello, puede decirse que la ubicación en el territorio de la oferta de alojamientos o complementaria parte de la existencia de recursos que permitan sustentar la actividad, pero también de otros aspectos que, en gran medida, han sido definidos como factores de localización efectivos, tal como han manifestado variados autores (Gunn, 1988; Ritchie, et al, 2005; Antón y González, 2011; Sánchez et al, 2013). Sin embargo, no siempre existe una correlación perfecta entre la presencia de atractivos y la implantación de la oferta, pues hay casos donde los alojamientos se han implantado por estar en un lugar de paso (oferta de carretera), por disponer de un volumen de población elevado en el contexto regional que se analiza, o bien por la apuesta que se ha realizado por dinamizar el sector a través de iniciativas tales como LEADER y PRODER. 
Sánchez Martín J. M., Rengifo Gallego J. I., Sánchez Rivero M. (2017): “Caracterización espacial del turismo en Extremadura mediante análisis de agrupamiento (Grouping Analysis). Un ensayo técnico”, GeoFocus (Artículos), $n^{\circ} 19$, p. 207-235. ISSN: 1578-5157. http://dx.doi.org/10.21138/GF.490

La enorme casuística que se detecta en algunos casos da lugar a que, como ocurre con cualquier técnica estadística, se produzcan ciertas indefiniciones, pues uno de los principales componentes del sistema turístico, la oferta de alojamientos, no siempre se guía por la coherencia. Lejos de ello, intervienen decisiones humanas, en ocasiones incomprensibles desde el rigor científico o la coherencia económica que se presupone a cualquier negocio, lo que ha dado lugar a que en zonas menos proclives al desarrollo turístico se haya implantado oferta alojativa. Dicha circunstancia, muy patente en el medio rural, fruto de las políticas expansivas de los programas de desarrollo fomentadas por la Unión Europea, provoca que se instalen alojamientos en zonas que no gozan del atractivo necesario. Ello, unido a la crisis económica y financiera que ha azotado a nuestro país y a la carencia de productos turísticos que identifiquen un territorio, entre otros motivos de peso, ha ocasionado, en los casos más extremos, el cierre de establecimientos como consecuencia de la baja rentabilidad económica.

Por otra parte, y tal vez fruto de la necesidad de establecer zonas de actuación amplias que trascienden al propio establecimiento o destino, ha proliferado en la literatura reciente la integración de las dimensiones geográficas al turismo. Éstas han puesto énfasis en la detección de patrones generales de distribución, aplicando para ello tanto consideraciones teóricas como empíricas, y cuya base metodológica ha partido de la aplicación de los más variados métodos de análisis locacionales (Yokeno, 1968; Haggett, 1976), hasta llegar a nuestros días, en los que se hace una utilización profusa de los análisis geoestadísticos (García-Palomares et al, 2015; Fang et al, 2015) como consecuencia de la incorporación de los mismos a los sistemas de información geográfica (SIG).

Fruto de esta relativa novedad en la aplicación de análisis geoestadísticos en la disciplina turística, surge este trabajo, que propone la aplicación del Análisis de Agrupaciones (Grouping Analysis) desde dos perspectivas diferenciadas: la primera, centrada en la omisión de restricciones espaciales, emulando a las agrupaciones realizadas por los métodos estadísticos tradicionales, cuya base está constituida por variables cuantitativas; la segunda, recurriendo a la proximidad espacial, no siempre exenta de problemas, pues los resultados son diferentes si se cambian la relaciones espaciales, algo obvio por otra parte. Por ello, y para mostrar esta complejidad, utilizamos las herramientas de asignación de clústers presentes en el módulo de estadística espacial del software ArcGIS 10.3.

Para el desarrollo de este análisis, se ha tomado como área de estudio la Comunidad Autónoma de Extremadura (España). Al realizar una aproximación al espacio turístico extremeño, se observa una gran diversidad de territorios turísticos, ya sea agrupados o diferenciados del resto, formando áreas o centros turísticos consolidados, tal y como sucede en las comarcas de La Vera o El Jerte, así como en Cáceres, Mérida o Trujillo. Sin embargo, prevalecen los territorios que se pueden denominar mixtos, en los que una parte de los municipios que los componen presenta un progreso turístico aceptable, mientras que en el resto de integrantes no se ha desarrollado la actividad. Nos enfrentamos, por lo tanto, a un espacio complejo, caracterizado por tener niveles de desarrollo turístico muy diferenciados, que presenta realidades u oportunidades diferentes como consecuencia de la disposición de los atractivos, de las facilidades de comunicación o de las características intrínsecas del propio espacio. Esto dificulta enormemente la siempre compleja tarea de planificar.

La mejor forma de desarrollar planes de turismo eficaces consiste en adaptarlos a las condiciones peculiares que ofrece cada territorio, para lo que se hace necesario conocer los componentes básicos sobre los que se sustenta la actividad en un territorio dado, pues cada espacio 
Sánchez Martín J. M., Rengifo Gallego J. I., Sánchez Rivero M. (2017): “Caracterización espacial del turismo en Extremadura mediante análisis de agrupamiento (Grouping Analysis). Un ensayo técnico”, GeoFocus (Artículos), $n^{\circ} 19$, p. 207-235. ISSN: 1578-5157. http://dx.doi.org/10.21138/GF.490

tiene sus fortalezas y sus debilidades en todos los aspectos que configuran su sistema turístico. Ahí radica la necesidad de conocer y analizar de forma detallada y específica cada uno de los espacios que componen el mosaico turístico de la zona de estudio.

Considerando estos antecedentes, se establece como punto de partida la hipótesis de que Extremadura está subdividida en numerosos micro-espacios turísticos. Éstos serían, aparentemente, diferentes a los utilizados para llevar a cabo políticas turísticas, basadas en delimitaciones que llegan a trascender la propia continuidad territorial, o tomando como base entidades territoriales que no reflejan la realidad del desarrollo turístico. Igualmente, se plantea que algunos de estos espacios turísticos pueden ser mucho más amplios al tener características comunes. Por todo ello, este trabajo busca la concreción territorial homogénea, para lo que opta por aplicar una técnica poco utilizada en la literatura, como es el análisis de agrupamiento (Grouping Analysis), exponiendo, además, la diferencia que muestra esta técnica al considerar una restricción espacial o centrándose exclusivamente en criterios cuantitativos.

El objetivo principal es determinar la existencia de zonas o territorios homogéneos en los que se pueden aplicar políticas turísticas comunes, en virtud de las características que los definen, teniendo éstas un marcado carácter territorial, y no solo numérico. Por lo tanto, la detección de estos territorios obedece a fundamentos turísticos y espaciales, independientemente de las agrupaciones, a veces forzadas, de municipios. Para alcanzar dicho objetivo, se apuesta por la aplicación de un análisis de clúster espacial, una aplicación eminentemente geoestadística en la línea de otros autores (Boers y Cottrell, 2007; Chancellor, 2008).

\section{2. Área de estudio}

Extremadura es una comunidad autónoma española de carácter periférico (figura 1), aunque no por ello exenta de atractivo para la práctica del turismo. Posee un patrimonio rico y variado, tanto en su concepción natural como cultural, que ha sido recogido y tratado en numerosas publicaciones, a escala regional (JUNTAEX, 2010), o a escalas locales (Sánchez, 1998; Rengifo, et al, 2013; Sánchez, et al, 2013; Sánchez, et al, 2014; Sánchez et al, 2017). Tan abundante patrimonio se sintetiza en dos vocaciones turísticas bien diferenciadas: por un lado, la orientada al turismo rural; por otro, la enfocada al turismo cultural. En buena parte del territorio, ambas vocaciones interactúan de forma complementaria, como consecuencia de la superposición de recursos en una misma área, a excepción de los grandes centros turísticos, donde predomina claramente una de las vocaciones. Así, existen municipios donde el atractivo preponderante se centra en la presencia de un paisaje de notable belleza, a veces protegido mediante alguna de las figuras específicas contempladas en la Ley 8/1998, de 26 de junio, de Conservación de la Naturaleza y Espacios Naturales de Extremadura, modificada por la Ley 9/2006, de 23 de diciembre. Estas áreas protegidas suman 1.257.787 Has., que vienen a representar el 30,6 \% de la Comunidad Autónoma. Destacan, entre otros espacios protegidos, el Parque Nacional de Monfragüe, el Geoparque Villuercas-Ibores-Jara y el Parque Natural Tajo-Internacional. A ellos se suman otros espacios que, pese a no gozar de protección, son enormemente atractivos para la demanda turística. Éste sería el caso de las zonas montañosas de mayor elevación, coincidentes con las estribaciones occidentales del Sistema Central, Los Montes de Toledo y Sierra Morena. Por las mismas discurren numerosos cursos de agua sobre los que se ha construido una infraestructura turística muy demandada en verano: las piscinas naturales, muy abundantes en el norte de Extremadura, aunque con un 
Sánchez Martín J. M., Rengifo Gallego J. I., Sánchez Rivero M. (2017): “Caracterización espacial del turismo en Extremadura mediante análisis de agrupamiento (Grouping Analysis). Un ensayo técnico”, GeoFocus (Artículos), $n^{\circ} 19$, p. 207-235. ISSN: 1578-5157. http://dx.doi.org/10.21138/GF.490

desarrollo más limitado o casi nulo en el resto del territorio. Por otra parte, existe un sobresaliente patrimonio histórico-artístico, con numerosos ejemplos de bienes que han alcanzado un nivel de protección elevado (Bienes de Interés Cultural -BIC-). Entre ellos se incluyen más de treinta Conjuntos Históricos. Asimismo, hay que mencionar la presencia de tres Bienes que forman parte del Listado del Patrimonio Mundial, como son Cáceres, Mérida y Guadalupe.

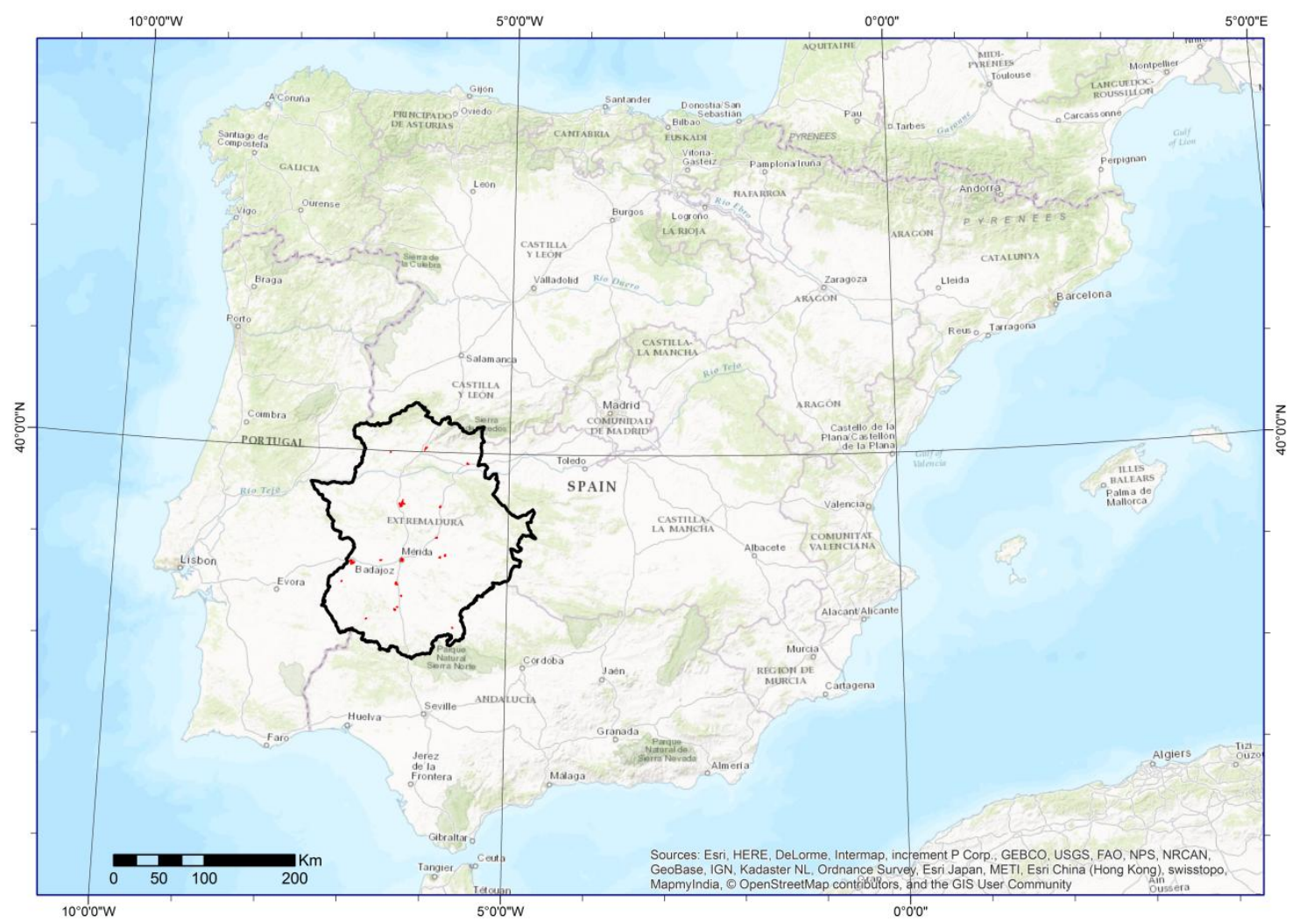

Figura 1: Localización del área de estudio

Fuente: Elaboración propia

Ambas tipologías de recursos pueden aparecer solapadas en el territorio, lo que contribuye a incrementar la capacidad de atracción de las áreas donde se produce esta circunstancia. No obstante, suele existir un tipo de atractivo determinante en el territorio que obedece a la vocación principal que posee.

A pesar de esta abundancia y variedad de atractivos, el desarrollo turístico de Extremadura se remonta a épocas recientes. En un principio se hallaba restringido a núcleos emblemáticos, como las ciudades de Cáceres, Mérida o Trujillo, a las que se añadían pequeños pueblos, como Baños de Montemayor, famoso desde comienzos del S.XVIII por su balneario, o Guadalupe, reconocido centro del turismo religioso. Por lo tanto, no eran numerosos los enclaves donde se practicaba el turismo, siendo éste de tipo cultural, básicamente, o vinculado a algún atractivo singular, como las aguas termales. Sin embargo, a partir de la década de los 90, comienza a despegar esta actividad, ampliando su oferta hacia la naturaleza y el mundo rural, hasta culminar hoy en día con otras apuestas, como el enoturismo, el oleoturismo o la observación de aves. 
Sánchez Martín J. M., Rengifo Gallego J. I., Sánchez Rivero M. (2017): “Caracterización espacial del turismo en Extremadura mediante análisis de agrupamiento (Grouping Analysis). Un ensayo técnico”, GeoFocus (Artículos), $n^{\circ} 19$, p. 207-235. ISSN: 1578-5157. http://dx.doi.org/10.21138/GF.490

Nos encontramos, por lo tanto, ante un panorama de crecimiento continuado en las propuestas y alternativas planteadas a los visitantes, que en parte de los casos implican la necesidad de realizar desplazamientos complementarios. De ahí deriva la importancia del territorio cercano. Esta circunstancia no es nueva, ni se circunscribe al turismo en exclusiva, ya que cualquier aspecto territorial presenta mayores relaciones con el ámbito más cercano que con el más alejado (Tobler, 1970).

A comienzos del presente siglo el desarrollo turístico comienza a tomar fuerza en la región, incrementándose y diversificándose la oferta, a la vez que aumenta su distribución por todo el territorio. Esta tendencia de crecimiento se aprecia en el número de alojamientos y, consecuentemente, en el de plazas (cuadro 1). No obstante, el aumento en la capacidad de hospedaje no tiene correspondencia directa con el crecimiento en el volumen de turistas o de pernoctaciones, lo que da lugar a un notable desajuste entre la oferta y la demanda. Dicho comportamiento antagónico entre sendas variables se ve agravado por el hecho de que la estancia media, así como el porcentaje medio de ocupación, no han experimentado variaciones significativas a lo largo de los años (INE). Ello confluye en una aparente crisis del sector, para cuya atenuación se han puesto en marcha diferentes políticas turísticas. Buena parte de las mismas se plantean como objetivo prioritario aumentar la estancia media, algo realmente difícil en un destino interior, en el que predominan los desplazamientos de fin de semana y puentes. Una interpretación más pausada de los datos refleja que tal vez sea más fácil y productivo recurrir a captar un mayor volumen de turistas, siquiera los fines de semana, con el fin de incrementar la rentabilidad de los establecimientos. Para ello se precisa disponer del territorio próximo como garantía de experiencia turística, pues en un espacio relativamente cercano es posible disfrutar de recursos muy variados, de gran riqueza y complementarios.

Cuadro 1: Evolución de oferta y demanda

\begin{tabular}{|c|r|r|r|r|r|}
\hline Años & Establecimientos & Plazas & Viajeros & Pernoctaciones & Pernoct/plazas \\
\hline 2001 & 643 & 19.773 & 1.197 .566 & 2.095 .977 & 106,0 \\
\hline 2002 & 632 & 20.866 & 1.121 .471 & 2.015 .945 & 96,6 \\
\hline 2003 & 696 & 22.810 & 1.152 .592 & 2.111 .233 & 92,6 \\
\hline 2004 & 725 & 23.845 & 1.182 .448 & 2.185 .793 & 91,7 \\
\hline 2005 & 774 & 24.984 & 1.171 .112 & 2.178 .929 & 87,2 \\
\hline 2006 & 853 & 26.552 & 1.321 .833 & 2.529 .365 & 95,3 \\
\hline 2007 & 926 & 27.488 & 1.409 .992 & 2.706 .404 & 98,5 \\
\hline 2008 & 1.011 & 29.294 & 1.369 .983 & 2.713 .575 & 92,6 \\
\hline 2009 & 1.114 & 30.918 & 1.395 .478 & 2.703 .638 & 87,4 \\
\hline 2010 & 1.143 & 31.852 & 1.383 .542 & 2.656 .024 & 83,4 \\
\hline 2011 & 1.224 & 33.641 & 1.535 .547 & 2.884 .547 & 85,7 \\
\hline 2012 & 1.308 & 34.760 & 1.452 .281 & 2.705 .020 & 77,8 \\
\hline 2013 & 1.359 & 35.362 & 1.423 .362 & 2.613 .183 & 73,9 \\
\hline 2014 & 1.365 & 34.614 & 1.481 .685 & 2.705 .760 & 78,2 \\
\hline 2015 & 1.358 & 34.885 & 1.623 .535 & 2.938 .656 & 84,2 \\
\hline Variación $2001-15$ & 715 & 15.112 & 425.969 & 842.679 & $-21,8$ \\
\hline & $111,2 \%$ & $76,4 \%$ & $35,6 \%$ & $40,2 \%$ & $-20,6 \%$ \\
\hline
\end{tabular}

Fuente: Elaboración propia sobre datos del INE (EOAH, EOTR, EOAC, EOAP) 
Sánchez Martín J. M., Rengifo Gallego J. I., Sánchez Rivero M. (2017): “Caracterización espacial del turismo en Extremadura mediante análisis de agrupamiento (Grouping Analysis). Un ensayo técnico”, GeoFocus (Artículos), $n^{\circ} 19$, p. 207-235. ISSN: 1578-5157. http://dx.doi.org/10.21138/GF.490

\section{Metodología}

\subsection{Fuentes de información.}

Para la elaboración de este trabajo se parte de la información suministrada por diferentes organismos oficiales (cuadro 2). En concreto, se toman como fuentes de datos las ofrecidas por el Instituto Nacional de Estadística (INE); la Dirección General de Turismo de la Junta de Extremadura y el Instituto Geográfico Nacional (IGN). La información que aporta cada uno de ellos es variada y contiene su propia metodología de elaboración y tratamiento de datos. De forma sucinta, se puede expresar que toda la información relativa a la evolución experimentada por el número de establecimientos, plazas, viajeros y pernoctaciones proviene del INE y hace referencia a todos los años contemplados en el análisis evolutivo, desde 2001 a 2015. En su nota metodológica se definen las variables incluidas en la Encuesta de Ocupación Hotelera (EOH), la Encuesta de Ocupación en Campamentos de Turismo (EOCT), la Encuesta de Ocupación en Apartamentos (EOA) y la Encuesta de Ocupación en Alojamiento de Turismo Rural (EOTR). Asimismo, se insiste en que la fuente de la que se obtienen los datos está conformada por las Consejerías de Turismo de cada comunidad autónoma. Cabe destacar, como aspecto significado, la consideración de los viajeros, ya que su definición está sujeta a la necesaria pernoctación. Por lo tanto, los datos de viajeros hacen referencia exclusivamente a los turistas, excluyendo por tanto a los excursionistas. Por su parte, de la Dirección General de Turismo procede la información relativa a los principales atractivos turísticos de la región no contemplados en la base de datos cartográfica. Al mismo tiempo, la base cartográfica utilizada es la proporcionada por el Instituto Geográfico Nacional (IGN), correspondiente a la Base Topográfica Nacional a escala 1:100.000. Esta escala de trabajo cuenta con una resolución de 20 metros e integra información geográfica suministrada por diversas fuentes oficiales.

Cuadro 2: Fuentes e información suministrada con nivel de agregación territorial

\begin{tabular}{|c|c|c|c|}
\hline Organización & Fuente & Información suministrada & Nivel \\
\hline \multirow{4}{*}{ INE } & EOH & Establecimientos & \multirow{4}{*}{$\begin{array}{l}\text { Comunidac } \\
\text { Autónoma }\end{array}$} \\
\hline & EOCT & Plazas & \\
\hline & EOA & Viajeros & \\
\hline & EOTR & Pernoctaciones & \\
\hline JUNTA DE EXTREMADURA & $\begin{array}{l}\text { Dirección } \\
\text { General } \\
\text { de } \\
\text { Turismo }\end{array}$ & $\begin{array}{l}\mathrm{N}^{\circ} \text { de alojamientos por tipo } \\
\mathrm{N}^{\circ} \text { de plazas según tipo de } \\
\text { alojamiento } \\
\text { Bienes de Interés Cultural } \\
\text { Otros recursos }\end{array}$ & Municipio \\
\hline IGN & BCN100 & $\begin{array}{l}\text { Divisiones administrativas y zonas } \\
\text { protegidas } \\
\text { Relieve } \\
\text { Hidrografía } \\
\text { Poblaciones y construcciones } \\
\text { Transportes }\end{array}$ & $\begin{array}{l}\text { Comunidad } \\
\text { Autónoma } \\
\text { Provincia } \\
\text { Municipio } \\
\text { Núcleo }\end{array}$ \\
\hline
\end{tabular}

Fuente: Elaboración propia 
Sánchez Martín J. M., Rengifo Gallego J. I., Sánchez Rivero M. (2017): “Caracterización espacial del turismo en Extremadura mediante análisis de agrupamiento (Grouping Analysis). Un ensayo técnico”, GeoFocus (Artículos), $n^{\circ} 19$, p. 207-235. ISSN: 1578-5157. http://dx.doi.org/10.21138/GF.490

El conjunto de las fuentes de datos usadas permitió generar una importante base de datos compuesta por más de 120 variables para cada uno de los 388 municipios de Extremadura. Sin embargo, este ingente volumen de información no podía considerarse para la aplicar la técnica de Análisis de Agrupaciones, debido a la propia complejidad de la misma, que recomienda utilizar un máximo de 15 variables para generar los grupos de forma eficaz.

Por ello, se decidió utilizar los parámetros principales que pueden intervenir de forma clara en la consecución de grupos de municipios turísticos homogéneos. Entre ellos están los atractivos, representados por la altitud máxima del término municipal, los Bienes de Interés Cultural, los museos y centros de interpretación, así como la superficie de agua embalsada, y la ocupada por el Parque Nacional y los Naturales, además de las zonas de baño y piscinas naturales que jalonan el territorio. Ello permite establecer una clara diferencia entre las zonas que presentan una clara vocación para el desarrollo de turismo rural y de naturaleza y las que lo hacen para el turismo cultural.

Al mismo tiempo, se han tenido en cuenta otros aspectos vinculados a la oferta turística, representada por el conjunto de alojamientos hoteleros y rurales, así como a la restauración. De igual modo, se ha optado por incluir la población residente, que, si bien no es un atractivo turístico, sí que resulta decisiva a la hora de explicar la implantación de determinada oferta.

\subsection{Técnicas de análisis.}

La componente territorial del turismo está fuera de toda duda desde la aparición del propio concepto, pues resulta innegable la trascendencia que tiene el conocimiento de los centros emisores y receptores para la correcta comprensión de la actividad y las repercusiones que conlleva su puesta en marcha. Sin embargo, pese a la necesidad de integrar el territorio como un elemento clave cuando se procede a caracterizar los municipios desde el punto de vista turístico, se observa cómo las relaciones espaciales quedan supeditadas a la consideración de otras variables, eminentemente cuantitativas y, en menor medida, cualitativas.

Son relativamente frecuentes los estudios que tratan de delimitar espacios turísticos homogéneos partiendo de criterios puramente cuantitativos, referidos a variables vinculadas a la presencia de atractivos, de oferta, sea ésta del tipo que fuere, o la existencia de otro tipo de facilidades turísticas. En cambio, hay pocas referencias cuando se trata de dotar a estos espacios de continuidad espacial, basada tanto en elementos cuantitativos como territoriales. Esta última consideración implica necesariamente la aplicación de técnicas geoestadísticas, a nuestro juicio enriquecedoras en cualquier tipo de análisis.

El objetivo de la asignación de clústers espaciales se centra en buscar grupos homogéneos y que, por tanto, tengan características parecidas en las variables utilizadas para el modelo. Su funcionamiento es relativamente complejo, aunque simplificándolo puede decirse que trata de encontrar agrupaciones naturales en los datos, buscando para ello valores de las variables parecidos entre todos los componentes del grupo y diferencias máximas en los registros con el resto de grupos. Es decir, busca semejanzas internas entre los datos y desigualdades externas, estableciendo una clara distinción entre los diferentes grupos que se generan. Además, si se opta por utilizar el 
Sánchez Martín J. M., Rengifo Gallego J. I., Sánchez Rivero M. (2017): “Caracterización espacial del turismo en Extremadura mediante análisis de agrupamiento (Grouping Analysis). Un ensayo técnico”, GeoFocus (Artículos), $n^{\circ} 19$, p. 207-235. ISSN: 1578-5157. http://dx.doi.org/10.21138/GF.490

criterio espacial como restricción, las agrupaciones deben reunir otro requisito más, la proximidad, determinada por el parámetro que se elija para su concreción.

Grosso modo, este tipo de análisis calcula el coeficiente de determinación existente para cada variable introducida $\left(\mathrm{R}^{2}\right)$, reflejando el valor que mantiene tras la realización de la agrupación oportuna y midiendo a la vez el grado de eficacia obtenido por los grupos creados. Tanto el valor $\mathrm{R}^{2}$ como el número de grupos varían en función de la restricción espacial impuesta, siendo uno de los elementos críticos y de mayor complejidad la determinación del número de agrupaciones óptimas. Para la obtención del mismo, se recurre al índice F-Statistic (Calinski y Harabasz, 1974), de reconocido uso en la literatura desde hace décadas.

Siguiendo este índice, la obtención del número de grupos óptimos se realiza mediante:

$$
\mathrm{R}^{2}=\frac{(\mathrm{TSS}-\mathrm{ESS})}{\mathrm{TSS}}
$$

donde TSS es la suma total de los cuadrados obtenidos para la variable considerada, siendo un reflejo de las diferencias entre los grupos; mientras ESS es la suma explicada de los cuadrados y muestra el nivel de similitud dentro de cada grupo. Sendos parámetros se calculan a través de la siguiente formulación:

$$
\begin{aligned}
& T S S=\sum_{i=1}^{n_{c}} \sum_{j=1}^{n_{i}} \sum_{v=1}^{n_{v}}\left(V_{i j}^{k}-\overrightarrow{V^{k}}\right)^{2} \\
& E S S=\sum_{i=1}^{n_{c}} \sum_{j=1}^{n_{i}} \sum_{v=1}^{n_{v}}\left(V_{i j}^{k}-\overrightarrow{V_{l}^{k}}\right)^{2}
\end{aligned}
$$

siendo $n$ el número de características; $n_{i}$ el número de características en el grupo $i ; n_{c}$ el número de clases o grupos; $n_{v i}$ el número de variables utilizadas, $V_{i j}^{k}$ el valor que adquiere la variable k-ésima en la característica j-ésima dentro del grupo i-ésimo, $\overrightarrow{V^{k}}$ el promedio de la variable k-ésima y $\overrightarrow{V_{l}^{k}}$ el valor medio de la variable k-ésima en el grupo $i$.

El software utilizado permite realizar el análisis de agrupamiento siguiendo diferentes tipos de relación espacial, si bien, el principal criterio discriminante es la omisión o la utilización de la restricción territorial. En el caso de recurrir a una restricción espacial, la casuística es considerable, debido a la gran variedad de combinaciones que existen. No obstante, las alternativas idóneas pasan por la consideración de las propias entidades territoriales que servirán de base para establecer las relaciones espaciales, ya que no serán similares en el caso de recurrir a los términos municipales o a los núcleos de población.

Cuando se toma como base de análisis territorial el término municipal, al ser entidades continuas en el territorio (véase figura 2), en contacto permanente entre ellas por tener ejes o nodos comunes, pueden utilizarse dichas características como criterio de restricción espacial. Sin embargo, cuando se esgrime como criterio espacial la existencia de ejes o vértices comunes se aprecia que el 


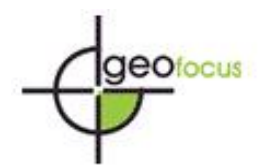

Sánchez Martín J. M., Rengifo Gallego J. I., Sánchez Rivero M. (2017): "Caracterización espacial del turismo en Extremadura mediante análisis de agrupamiento (Grouping Analysis). Un ensayo técnico”, GeoFocus (Artículos), $n^{\circ} 19$, p. 207-235. ISSN: 1578-5157. http://dx.doi.org/10.21138/GF.490

número de entidades que se vincularían al análisis es muy variado. Esto se pone de relieve en términos municipales como Cáceres, colindante con otros 27 términos, aunque hay otros, como Valencia de Alcántara, que sólo hace frontera con 8 , e incluso casos como el de Cedillo, que tan solo está en contacto con otro municipio.

Naturalmente, no parece acertado desde un punto de vista metodológico utilizar como unidad de análisis el término municipal, ni siquiera seleccionando el número de vecinos que se quiere utilizar como parámetro de agrupación.
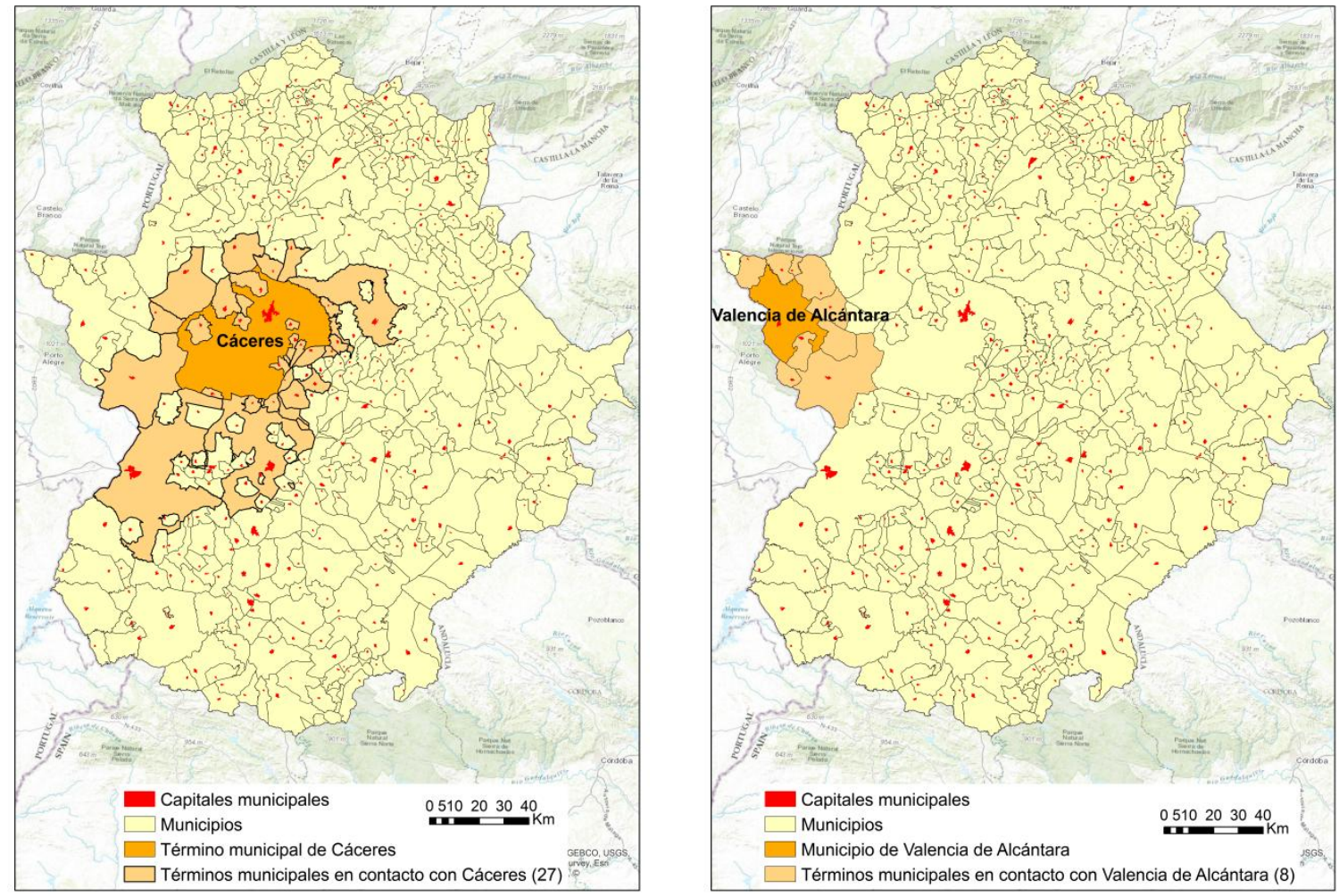

Figura 2: Utilización de Términos municipales como restricción territorial Fuente: Elaboración propia

En cambio, cuando la unidad de análisis territorial es la capital de término municipal y, más concretamente los centroides internos de las mismas (véase figura 3), las posibilidades se reducen a una triangulación de Delaunay o al uso del número de vecinos, aparte de la omisión del criterio espacial.

El método de triangulación de Delaunay genera una poligonación de Thiessen cuyos vértices son los centroides. De ese modo, siempre existirán ejes comunes entre ellos, aunque es posible que algunos no tengan continuidad espacial. Por ello, dado que el objetivo es crear polígonos nuevos para obtener este tipo de restricción territorial, tal vez sea más apropiado recurrir al número de vecinos próximos. Este método seleccionaría los vecinos más cercamos teniendo en cuenta la distancia euclidiana formada por los centroides en función del número de casos que se determinen. 
Sánchez Martín J. M., Rengifo Gallego J. I., Sánchez Rivero M. (2017): “Caracterización espacial del turismo en Extremadura mediante análisis de agrupamiento (Grouping Analysis). Un ensayo técnico”, GeoFocus (Artículos), $n^{\circ} 19$, p. 207-235. ISSN: 1578-5157. http://dx.doi.org/10.21138/GF.490
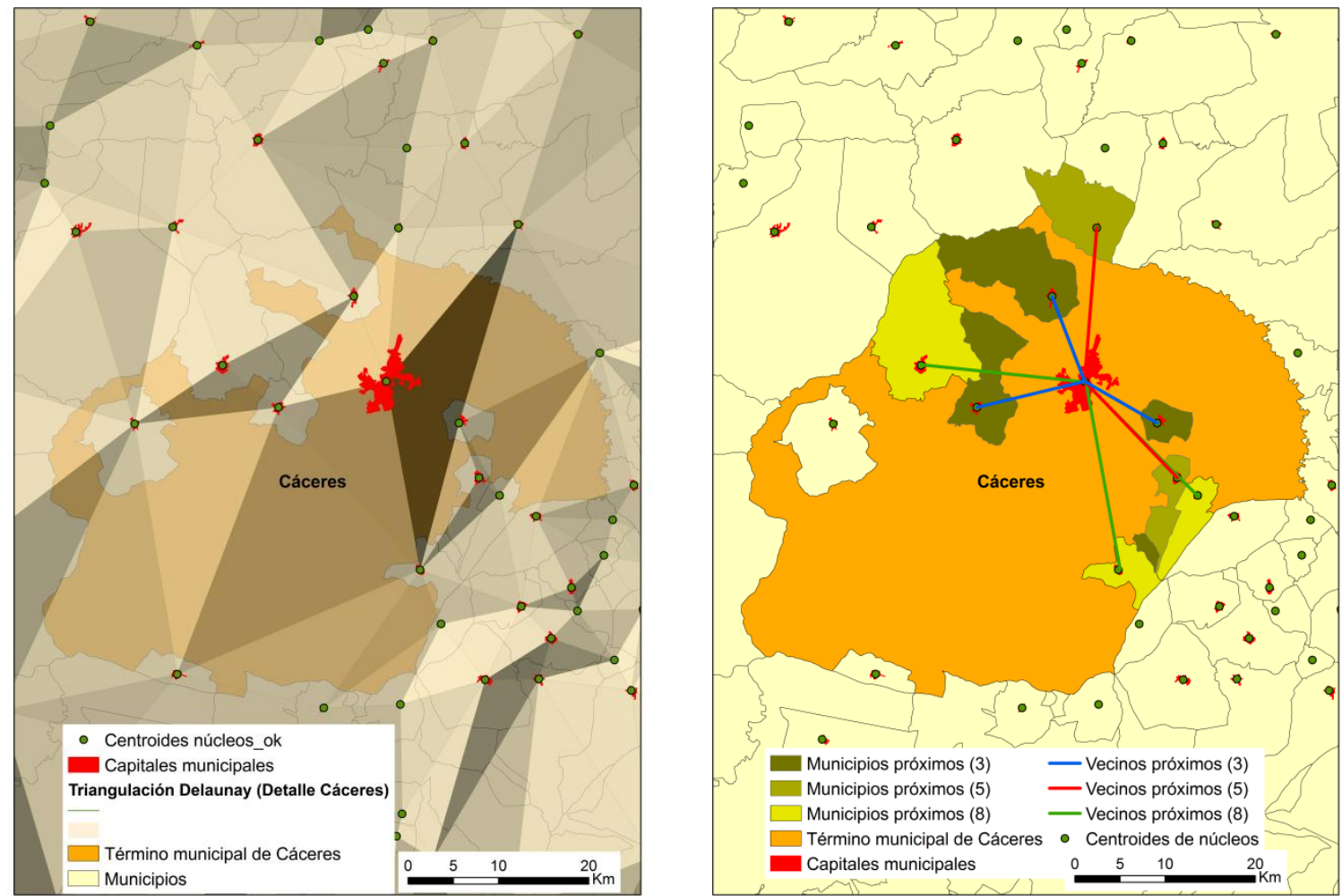

Figura 3: Utilización de capitales de término municipal como restricción territorial: triangulación de Delaunay (izquierda) y vecinos próximos (derecha)

Fuente: Elaboración propia

A tenor de lo expuesto, y considerando la peculiar distribución municipal de Extremadura, se estima que, desde un punto de vista metodológico, lo más acertado es recurrir a la utilización de las capitales de término municipal como entidad territorial. Por ello, la aplicación de la técnica se ciñe a mostrar los resultados que se alcanzan teniendo en cuenta las diferentes relaciones espaciales que permite la utilización de dichas entidades, que han sido simplificadas mediante los centroides internos de las capitales de los términos municipales.

El análisis de agrupamiento construye un grafo de conectividad en el que se representan las relaciones de vecindad entre las entidades, generando un árbol de expansión mínima. Éste será reflejo de la estructura espacial de los núcleos y de las variables vinculadas al análisis. Posteriormente, se determina el lugar idóneo para dividir el árbol genérico en dos grupos que contengan la mejor solución posible. Tras ello, se procede a determinar cuál de las dos agrupaciones obtenidas podrá seguir dividiéndose, para así obtener un tercer grupo y así sucesivamente, hasta alcanzar el número óptimo de grupos en que puede dividirse (véase figura 4).

Para ilustrar su funcionamiento, hemos elegido como caso específico uno de los utilizados en nuestro análisis, obtenido sin restricciones espaciales. Como se observa en este caso, al realizar el 
Sánchez Martín J. M., Rengifo Gallego J. I., Sánchez Rivero M. (2017): “Caracterización espacial del turismo en Extremadura mediante análisis de agrupamiento (Grouping Analysis). Un ensayo técnico”, GeoFocus (Artículos), $n^{\circ} 19$, p. 207-235. ISSN: 1578-5157. http://dx.doi.org/10.21138/GF.490

primer árbol de expansión mínima, hay dos grupos poco definidos aparentemente, aunque al realizar la agrupación definitiva y recomendada por el índice F-Statistic, el grupo 1, se ha escindido y queda compuesto exclusivamente por Cáceres, Badajoz y Mérida. Esta división da lugar a que el resto de núcleos adopte una agrupación nueva debido a que han sido eliminados del mismo los núcleos que tienen unas características más urbanas, al ser los núcleos más poblados y, por lo tanto, los más análogos entre sí, con grandes diferencias frente al resto de municipios analizados. Se observa que aparecen zonas muy representativas desde el punto de vista turístico, como es el caso de La Vera, el Valle del Jerte, Las Hurdes o la Sierra de Gata.
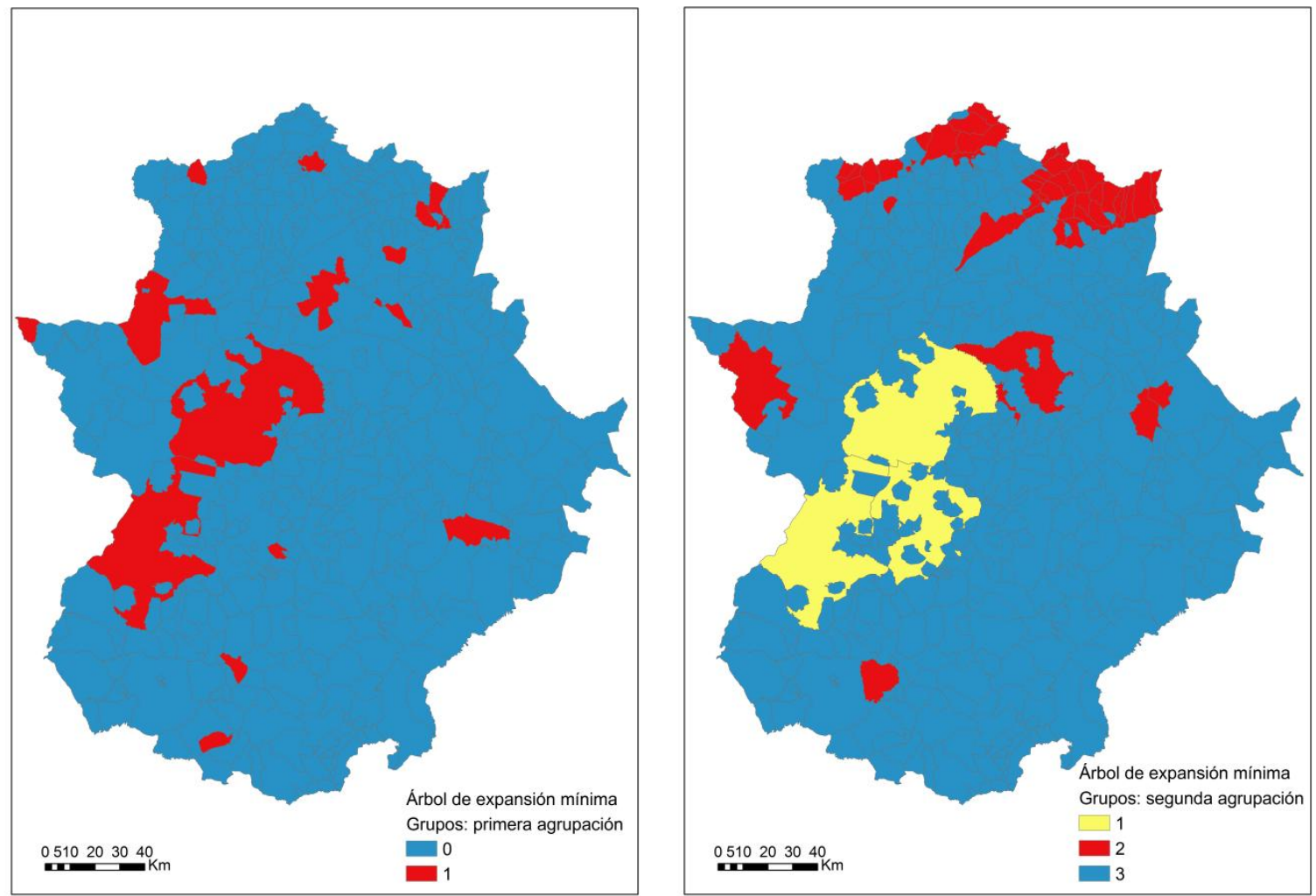

Figura 4: Representación territorial del árbol de expansión mínima (ejemplo ilustrativo sin restricción espacial y número de grupos optimizado mediante F-Statistic)

Fuente: Elaboración propia

Por otra parte, debido a que la técnica en sí ofrece múltiples posibilidades de análisis, se ha optado por aplicarla sobre el mismo conjunto de variables y entidades, con el objetivo fundamental de comprobar el comportamiento y los resultados que ofrecen tomando como referencia las diferentes relaciones espaciales que permiten las entidades seleccionadas. En concreto se aplican tres tipos de relación espacial, entre las que se encuentran: i) la propia omisión de la restricción espacial, es decir, tan solo se considera la relación de los datos asociados al propio análisis; ii) la utilización de una relación espacial basada en la triangulación de Delaunay, que convierte las entidades analizadas en una poligonación de Thiessen que determina la relación de vecindad; y iii) la utilización del número de vecinos próximos. 
Sánchez Martín J. M., Rengifo Gallego J. I., Sánchez Rivero M. (2017): “Caracterización espacial del turismo en Extremadura mediante análisis de agrupamiento (Grouping Analysis). Un ensayo técnico”, GeoFocus (Artículos), $n^{\circ} 19$, p. 207-235. ISSN: 1578-5157. http://dx.doi.org/10.21138/GF.490

Tanto a la hora de establecer la triangulación de Delaunay como los vecinos próximos, se ha tomado como referencia la distancia euclidinana entre los 388 centroides de los núcleos de población, correspondientes exclusivamente a las capitales de los términos municipales. Sin embargo, para mejorar la visibilidad cartográfica de los resultados, los datos se encuentran representados sobre el término municipal.

\section{Descripción y análisis de resultados.}

La aplicación del análisis de agrupamiento muestra resultados dispares dependiendo de la utilización del criterio complementario de restricción territorial que se utilice o al prescindir del mismo. Al omitir la limitación espacial, tan solo intervienen los valores que registran las variables consideradas, por lo que los resultados expresan únicamente grupos que tienen características parecidas. Éstos pueden aparecer alejados y sin formar un espacio homogéneo desde el punto de vista territorial, algo similar a lo que puede obtenerse mediante la utilización de otras técnicas de carácter cuantitativo exclusivamente. Sin embargo, cuando se impone una restricción espacial, se conjuga esta homogeneidad en las variables con la proximidad territorial, de tal modo que se obtienen áreas de mayor amplitud que disponen de características muy similares.

A nuestro juicio, los resultados que ofrece cada forma de obtener los grupos son muy reveladores, puesto que, si nos centramos exclusivamente en los criterios numéricos, sin otorgar ningún peso específico a la componente espacial, se obtienen 3 grupos óptimos bien diferenciados. Por su parte, si se apuesta por configurar un modelo de agrupaciones tomando como referencia la triangulación de Delaunay, se establecen hasta 9 grupos. Finalmente, si se considera el criterio territorial de vecindad como complemento al numérico definido por las variables, se definen 14 grupos bien perfilados. Resulta obvio que los efectos son bastante diferentes, pues en el segundo y en el tercer caso se utiliza como elemento vertebrador el propio territorio circundante.

Junto a ello, también cabe mencionar que, dependiendo del criterio territorial seleccionado, la contribución de cada variable al grupo, representada mediante $\mathrm{R}^{2}$, es diferente (véase cuadro 3 ). Este coeficiente mide la eficiencia del propio modelo configurado para la creación de estos conjuntos homogéneos. Así, cuando se recurre exclusivamente a los parámetros cuantitativos de las variables consideradas y omitiendo cualquier tipo de restricción espacial, obtenemos que $\mathrm{R}^{2}$ promediado para el conjunto de variables es de 0,446. En cambio, cuando se recurre a la consideración de la restricción espacial, impuesta por la triangulación de Delaunay y por los vecinos próximos, se obtienen mejores resultados de $\mathrm{R}^{2}$ promedios: 0,560 y 0,689 , respectivamente. 
Sánchez Martín J. M., Rengifo Gallego J. I., Sánchez Rivero M. (2017): “Caracterización espacial del turismo en Extremadura mediante análisis de agrupamiento (Grouping Analysis). Un ensayo técnico”, GeoFocus (Artículos), $n^{\circ} 19$, p. 207-235. ISSN: 1578-5157. http://dx.doi.org/10.21138/GF.490

Cuadro 3: Resumen comparativo de ajustes del grupo y variables

\begin{tabular}{|l|c|c|c|c|c|c|}
\hline \multicolumn{1}{|c|}{ Variable } & $\begin{array}{c}\mathbf{( A )} \mathbf{R}^{\mathbf{2}} \text { Sin } \\
\text { restricción } \\
\text { espacial }\end{array}$ & $\begin{array}{c}\mathbf{( B )} \mathbf{R}^{\mathbf{2}} \\
\text { triang. } \\
\text { Delaunay }\end{array}$ & $\begin{array}{c}\text { (C) } \mathbf{R}^{\mathbf{2}} \\
\text { vecino } \\
\text { próx. }\end{array}$ & $\begin{array}{c}\text { (C-A) } \mathbf{R}^{\mathbf{2}} \\
\mathbf{D i f .}\end{array}$ & $\begin{array}{c}\text { (B-A) } \mathbf{R}^{\mathbf{2}} \\
\mathbf{D i f} .\end{array}$ & (C-B) $\mathbf{R}^{\mathbf{2} \text { Dif. }}$ \\
\hline $\begin{array}{l}\text { Altitud } \\
\text { máxima }\end{array}$ & 0,496 & 0,387 & 0,607 & 0,111 & $-0,109$ & 0,221 \\
\hline $\begin{array}{l}\text { Bien de } \\
\text { Interés } \\
\text { Cultural }\end{array}$ & 0,661 & 0,756 & 0,789 & 0,128 & 0,095 & 0,033 \\
\hline $\begin{array}{l}\text { Museos y } \\
\text { centros de } \\
\text { interpretación }\end{array}$ & 0,658 & 0,668 & 0,770 & 0,112 & 0,010 & 0,102 \\
\hline $\begin{array}{l}\text { Plazas en } \\
\text { alojamientos } \\
\text { hoteleros }\end{array}$ & 0,786 & 0,782 & 0,824 & 0,039 & $-0,003$ & 0,042 \\
\hline $\begin{array}{l}\text { Plazas en } \\
\text { alojamientos } \\
\text { rurales }\end{array}$ & 0,385 & 0,268 & 0,405 & 0,020 & $-0,117$ & 0,137 \\
\hline $\begin{array}{l}\text { Plazas en } \\
\text { Restaurantes }\end{array}$ & 0,756 & 0,791 & 0,824 & 0,068 & 0,035 & 0,033 \\
\hline Población & 0,733 & 0,838 & 0,878 & 0,145 & 0,105 & 0,040 \\
\hline $\begin{array}{l}\text { Superficie de } \\
\text { embalses }\end{array}$ & 0,026 & 0,091 & 0,375 & 0,349 & 0,065 & 0,283 \\
\hline $\begin{array}{l}\text { Superficie en } \\
\text { parque } \\
\text { Nacional }\end{array}$ & 0,128 & 0,884 & 0,878 & 0,749 & 0,756 & $-0,006$ \\
\hline $\begin{array}{l}\text { Superficie en } \\
\text { parque } \\
\text { natural }\end{array}$ & 0,002 & 0,408 & 0,524 & 0,522 & 0,406 & 0,116 \\
\hline $\begin{array}{l}\text { Zonas de } \\
\text { baño/piscinas } \\
\text { naturales }\end{array}$ & 0,278 & 0,285 & 0,701 & 0,423 & 0,007 & 0,416 \\
\hline Promedio & 0,446 & 0,560 & 0,689 & 0,242 & 0,155 & 0,130 \\
\hline
\end{tabular}

Fuente: Elaboración propia

\subsection{Análisis de grupos sin restricción espacial.}

El resultado que ofrece el análisis de agrupamiento cuando se omite la restricción espacial presenta un nivel de eficiencia relativamente reducido. Si bien como promedio se obtiene un $\mathrm{R}^{2}$ de algo más de 0,466 , no se maximiza la representatividad de todas las variables utilizadas para el análisis, ya que buena parte de las mismas distan mucho de alcanzar un $\mathrm{R}^{2}$ de 0,500 . Llama la atención que los atractivos naturales apenas si son eficientes a la hora de configurar los grupos, a excepción de la altitud máxima, que se alcanza en el término municipal sobre el que se asienta el 
Sánchez Martín J. M., Rengifo Gallego J. I., Sánchez Rivero M. (2017): “Caracterización espacial del turismo en Extremadura mediante análisis de agrupamiento (Grouping Analysis). Un ensayo técnico”, GeoFocus (Artículos), $n^{\circ} 19$, p. 207-235. ISSN: 1578-5157. http://dx.doi.org/10.21138/GF.490

núcleo analizado. Ello puede observarse en la escasa contribución que tienen los espacios protegidos, los embalses e incluso un equipamiento fundamental para una parte de los turistas, las zonas de baño. Aparte de ello, esa misma situación afecta a las plazas en alojamientos rurales, uno de los pilares fundamentales del sistema turístico de Extremadura.

En cambio, el modelo sobre el que se realizan las agrupaciones es sensiblemente más eficiente cuando se analizan variables vinculadas al turismo cultural, pues aparecen con un $\mathrm{R}^{2}$ elevado los principales atractivos culturales, así como los equipamientos que con ellos se suelen asimilar. Por otra parte, tanto los BIC, como los museos y centros de interpretación superan un $\mathrm{R}^{2}$ de 0,650. Además, variables como las plazas en alojamientos hoteleros o en restaurantes y el volumen de población superan el 0,700 .

Por todo ello, el modelo de 3 grupos obtenido sin restricción espacial indicaría, a priori, que puede presentar serias limitaciones a la hora de interpretar los grupos que se podrían identificar con el turismo cultural y urbano y con el turismo rural. No obstante, se impone un análisis más pausado de cada uno de los grupos, donde se interprete la contribución que hace cada variable a cada uno de ellos (véase cuadro 4) y su distribución sobre el territorio (véase figura 5).

Cuadro 4: Contribución de variables a cada grupo sin restricción espacia
\begin{tabular}{|l|c|c|c|c|}
\hline Variable & Gr. 1 & Gr. 2 & Gr. 3 & $\mathbf{R}^{\mathbf{2}}$ \\
\hline Altitud máxima & 0,063 & 0,669 & 0,746 & 0,496 \\
\hline Bien de Interés Cultural & 0,667 & 0,185 & 0,333 & 0,661 \\
\hline Museos y centros de interpretación & 0,400 & 0,200 & 0,500 & 0,658 \\
\hline Plazas en alojamientos hoteleros & 0,191 & 0,279 & 0,404 & 0,786 \\
\hline Plazas en alojamientos rurales & 0,099 & 0,434 & 0,970 & 0,385 \\
\hline Plazas en Restaurantes & 0,434 & 0,259 & 0,281 & 0,756 \\
\hline Población & 0,607 & 0,247 & 0,271 & 0,733 \\
\hline Superficie de embalses & 0,533 & 1,000 & 0,396 & 0,026 \\
\hline Superficie en parque Nacional & 0,000 & 1,000 & 0,000 & 0,128 \\
\hline Superficie en parque natural & 0,990 & 1,000 & 0,250 & 0,002 \\
\hline Zonas de baño/piscinas naturales & 0,143 & 0,143 & 1,000 & 0,278 \\
\hline
\end{tabular}

Fuente: Elaboración propia

En este sentido, cabe señalar que el 1 está compuesto por aquellos núcleos que cuentan con parque natural, BIC, están bastante poblados, poseen una superficie de embalses importante y numerosas plazas en restaurantes. Lo conforman las tres principales ciudades: Badajoz, Cáceres y Mérida.

El grupo 2 enfatiza el peso que tienen las variables naturales, destacando la superficie en parques naturales y nacionales, así como la ocupada por embalses, a las que se añade además una altitud máxima elevada y, en menor medida, las plazas en alojamientos rurales. A nivel territorial coincide con el norte de la provincia de Cáceres, aunque, dada su configuración, también aparece 
Sánchez Martín J. M., Rengifo Gallego J. I., Sánchez Rivero M. (2017): “Caracterización espacial del turismo en Extremadura mediante análisis de agrupamiento (Grouping Analysis). Un ensayo técnico”, GeoFocus (Artículos), $n^{\circ} 19$, p. 207-235. ISSN: 1578-5157. http://dx.doi.org/10.21138/GF.490

representado en núcleos con una clara vinculación al turismo cultural, como sucede en Trujillo o Jerez de los Caballeros.

Por último, el grupo 3 está compuesto por los núcleos que disponen de zonas de baño, alojamientos rurales o una elevada altitud en su término municipal, aunque también aparecen significativas relaciones con otras variables, no tan vinculadas al turismo rural. A esta complejidad se añade el hecho de que ocupa la mayor parte del territorio, no siempre coincidente con el desarrollo del turismo rural.

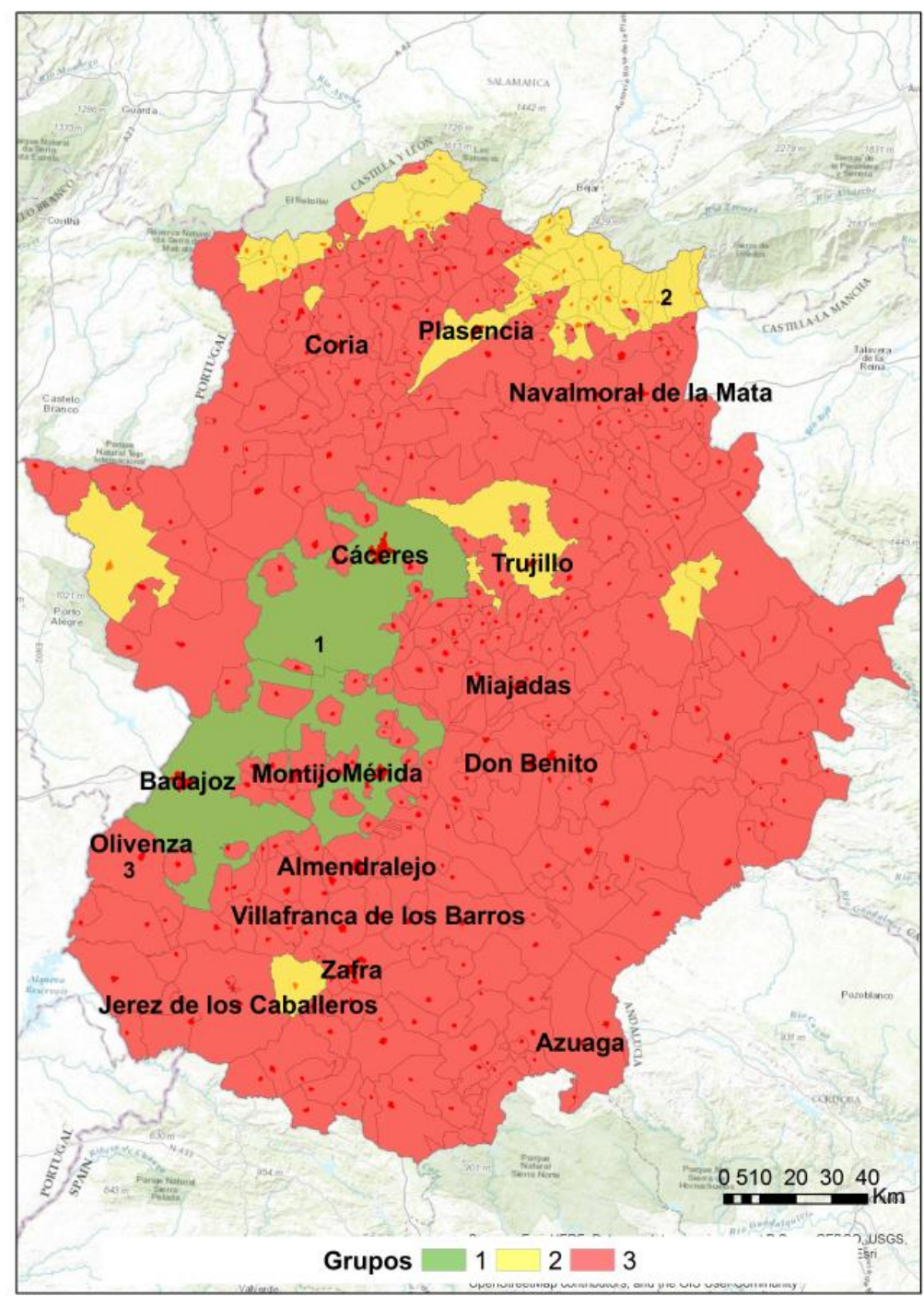

Figura 5: Análisis de grupos sin restricción espacial

Fuente: Elaboración propia 
Sánchez Martín J. M., Rengifo Gallego J. I., Sánchez Rivero M. (2017): “Caracterización espacial del turismo en Extremadura mediante análisis de agrupamiento (Grouping Analysis). Un ensayo técnico”, GeoFocus (Artículos), $n^{\circ} 19$, p. 207-235. ISSN: 1578-5157. http://dx.doi.org/10.21138/GF.490

\subsection{Análisis de grupos con restricción espacial.}

\subsubsection{Triangulación de Delaunay.}

La agrupación espacial obtenida tras la aplicación de la triangulación de Delaunay sobre los centroides de 3 núcleos de población, ofrece resultados más complejos que cuando se prescinde de la restricción espacial, consecuencia lógica tras la incorporación de un criterio de proximidad espacial a la de por sí compleja relación entre las variables consideradas. A pesar de ello, se observa que el promedio de ajuste del modelo, cifrado en un $\mathrm{R}^{2}$ de 0,56 , es aceptable en un sistema que analiza 388 casos, a lo que se une el hecho de que numerosas variables están representadas por encima de 0,650 . Por otra parte, no se puede pasar por alto que se crean 9 grupos relativamente homogéneos, tanto en lo referido a parámetros turísticos como a integridad territorial.

Las variables que más contribuyen a la configuración de los grupos son aquéllas que se vinculan a la práctica del turismo cultural y urbano. Entre ellas destacan la población, los alojamientos hoteleros y las plazas de restauración, y en menor medida, los BIC, museos, e incluso la superficie de Parque Nacional.

Cuando se profundiza en la contribución que realizan las variables a cada grupo (véase cuadro 5), se observa el interés que posee la técnica, sobre todo si se tiene en consideración cómo discrimina algunos grupos. Tal es el caso de los grupos 1 y 9 , cuya diferencia fundamental pasa por determinar aquéllos que, aun teniendo un recurso común de la categoría de Parque Nacional, se diferencian por la presencia de alojamientos rurales y zonas embalsadas. Es decir, escinde un grupo debido a su localización más alejada de las áreas clave del espacio protegido. Como consecuencia de ello, éstas no serían zonas propicias para la instalación de la oferta de alojamientos rurales, que sí están localizados en la zona más representativa, en la que, además, confluye una importante lámina de agua embalsada.

Los grupos 4 y 7 presentan una situación similar, pues ambos muestran un claro enfoque hacia el turismo rural, pero con sutiles diferencias que vienen marcadas por la presencia de complementos culturales, como museos y zonas con embalses.

Por su parte, los grupos 2, 3 y 6 no tienen un peso específico importante dentro de ninguna variable, pues debido a sus peculiaridades, no es posible establecer una relación espacial entre ellos, al estar solo implicados 2 municipios, en vez de los necesarios para la configuración del grupo. El grupo 5 apenas está representado por los núcleos del entorno del Parque Natural de Cornalvo, en las proximidades de Mérida Finalmente, el grupo 8 está poco definido, ya que no existe homogeneidad conformada por el número de núcleos que hemos determinado en la restricción espacial. 
Sánchez Martín J. M., Rengifo Gallego J. I., Sánchez Rivero M. (2017): “Caracterización espacial del turismo en Extremadura mediante análisis de agrupamiento (Grouping Analysis). Un ensayo técnico”, GeoFocus (Artículos), $n^{\circ} 19$, p. 207-235. ISSN: 1578-5157. http://dx.doi.org/10.21138/GF.490

Cuadro 5: Contribución de variables a cada grupo con triangulación de Delaunay

\begin{tabular}{|l|c|c|c|c|c|c|c|c|c|c|}
\hline Variable & Gr. 1 & Gr. 2 & Gr. 3 & Gr. 4 & Gr. 5 & Gr. 6 & Gr. 7 & Gr. 8 & Gr. 9 & $\mathbf{R}^{2}$ \\
\hline Altitud máxima & 0,215 & 0,000 & 0,000 & 0,619 & 0,068 & 0,000 & 0,814 & 0,763 & 0,175 & 0,387 \\
\hline Bien de Interés Cultural & 0,111 & 0,000 & 0,000 & 0,111 & 0,037 & 0,000 & 0,074 & 0,333 & 0,074 & 0,756 \\
\hline $\begin{array}{l}\text { Museos y centros de } \\
\text { interpretación }\end{array}$ & 0,000 & 0,000 & 0,000 & 0,200 & 0,000 & 0,000 & 0,300 & 0,500 & 0,100 & 0,668 \\
\hline $\begin{array}{l}\text { Plazas en alojamientos } \\
\text { hoteleros }\end{array}$ & 0,033 & 0,000 & 0,000 & 0,099 & 0,038 & 0,000 & 0,194 & 0,404 & 0,089 & 0,782 \\
\hline $\begin{array}{l}\text { Plazas en alojamientos } \\
\text { rurales }\end{array}$ & 0,030 & 0,000 & 0,000 & 0,783 & 0,072 & 0,000 & 1,000 & 0,757 & 0,385 & 0,268 \\
\hline Plazas en Restaurantes & 0,041 & 0,000 & 0,000 & 0,077 & 0,011 & 0,000 & 0,066 & 0,281 & 0,018 & 0,791 \\
\hline Población & 0,010 & 0,000 & 0,000 & 0,042 & 0,003 & 0,000 & 0,026 & 0,272 & 0,010 & 0,838 \\
\hline Superficie de embalses & 0,050 & 0,000 & 0,000 & 0,000 & 0,000 & 0,000 & 0,361 & 1,000 & 0,476 & 0,091 \\
\hline $\begin{array}{l}\text { Superficie en parque } \\
\text { Nacional }\end{array}$ & 0,292 & 0,000 & 0,000 & 0,000 & 0,000 & 0,000 & 0,000 & 0,138 & 0,543 & 0,884 \\
\hline $\begin{array}{l}\text { Superficie en parque } \\
\text { natural }\end{array}$ & 0,000 & 0,000 & 0,000 & 0,000 & 0,114 & 0,000 & 0,000 & 1,000 & 0,000 & 0,408 \\
\hline $\begin{array}{l}\text { Zonas de baño/piscinas } \\
\text { naturales }\end{array}$ & 0,000 & 0,000 & 0,000 & 1,000 & 0,000 & 0,000 & 1,000 & 0,429 & 0,000 & 0,285 \\
\hline
\end{tabular}

Fuente: Elaboración propia

El análisis territorial de los grupos muestra una configuración interesante en el caso que atañe al turismo cultural y urbano, representado por las 3 grandes ciudades, al mismo tiempo que muestra una agrupación efectiva importante sobre el Parque Nacional de Monfragüe. Sin embargo, cuando se analizan los grupos donde se ha desarrollado más el turismo rural, el ajuste no es bueno, como lo prueba el hecho de que hay zonas peculiares, como la Sierra de Gata, que no aparecen. Además de ello, la amplia distribución del Grupo 8, muy heterogéneo, encubriría posibles grupos específicos, debido a sus características especiales, como sucedería en el caso de Trujillo, uno de los grandes destinos del turismo cultural en el contexto extremeño, o zonas próximas a los grandes embalses de Extremadura. 
Sánchez Martín J. M., Rengifo Gallego J. I., Sánchez Rivero M. (2017): “Caracterización espacial del turismo en Extremadura mediante análisis de agrupamiento (Grouping Analysis). Un ensayo técnico”, GeoFocus (Artículos), $n^{\circ} 19$, p. 207-235. ISSN: 1578-5157. http://dx.doi.org/10.21138/GF.490

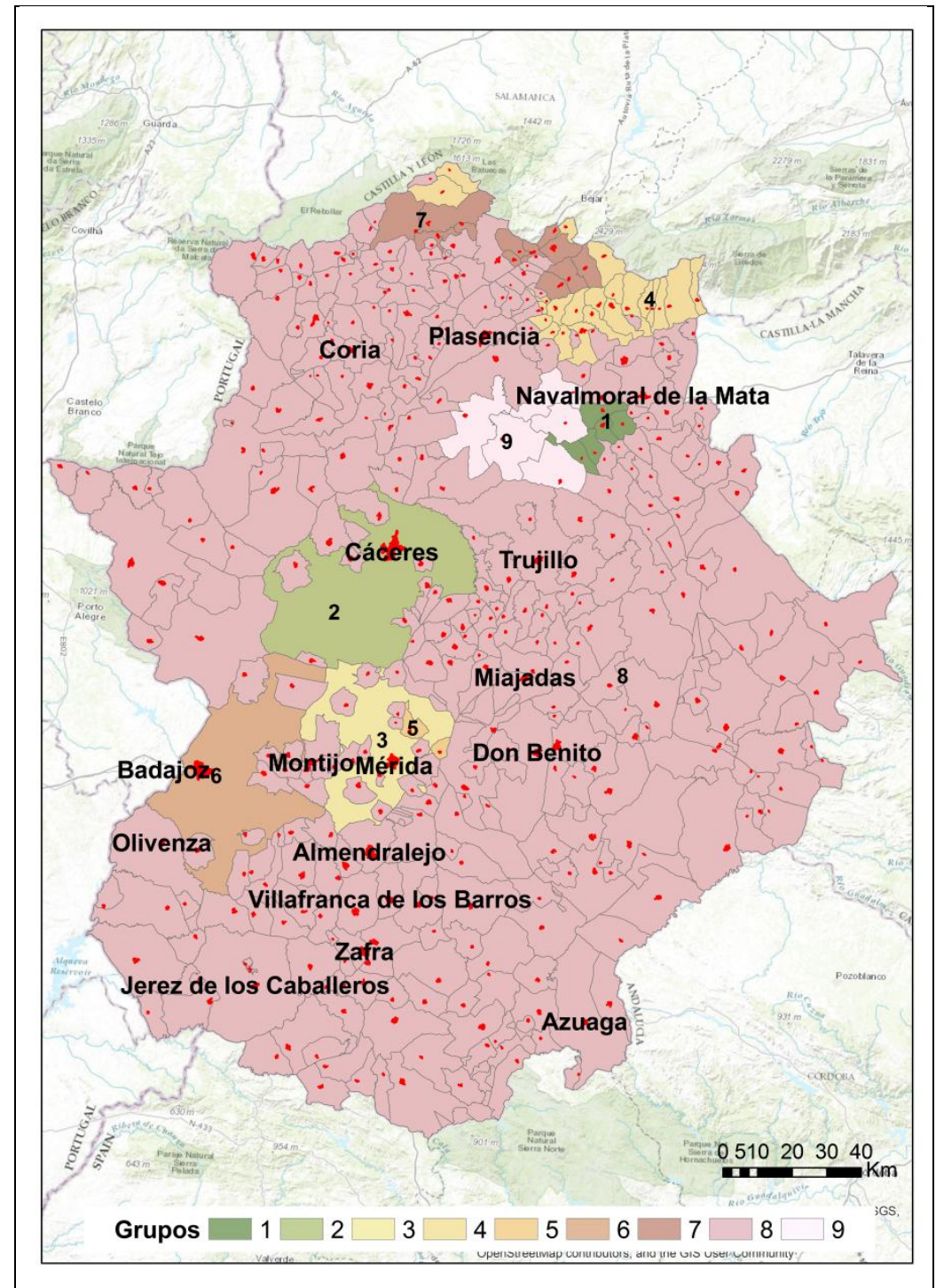

Figura 6: Análisis de grupos con triangulación de Delaunay

Fuente: Elaboración propia

\subsubsection{Vecinos próximos.}

Cuando se recurre a la utilización del criterio espacial tomando como base la relación que se establece entre los 3 vecinos más cercanos entre sí, mediante la distancia euclidiana sobre los centroides de los núcleos de población, la situación cambia radicalmente, pues aparecen 14 grupos bien definidos y delimitados en el territorio. 
Sánchez Martín J. M., Rengifo Gallego J. I., Sánchez Rivero M. (2017): “Caracterización espacial del turismo en Extremadura mediante análisis de agrupamiento (Grouping Analysis). Un ensayo técnico”, GeoFocus (Artículos), $n^{\circ} 19$, p. 207-235. ISSN: 1578-5157. http://dx.doi.org/10.21138/GF.490

La contribución que hace cada una las variables consideradas a la explicación del modelo es sensiblemente mayor que la obtenida mediante la triangulación de Delaunay o la omisión de la restricción espacial. Los parámetros que presentan menor $\mathrm{R}^{2}$ son la superficie de embalses y las plazas en alojamientos rurales, así como la superficie en parque natural, aunque los valores son siempre significativos en una muestra de 388 casos. Prueba de ello es que la contribución inferior se sitúa en 0,375. A ello se debe añadir que, en numerosas variables, se llega a superar un $R^{2}$ de 0,700 , e incluso 0,800 , circunstancia que nos induce a considerar la bondad del modelo.

Junto a ello, se observa que la contribución que hace cada variable a los diferentes grupos es muy diversa (véase cuadro 6), si bien el elevado peso de algunas de ellas en varios grupos refleja la complejidad de los modelos. No obstante, se observa que las más complejas se refieren al volumen de plazas de alojamiento, ya sea hotelero o rural, como consecuencia de que la implantación de este tipo de oferta abarca territorios muy diversos. También cabe destacar que la presencia de alojamientos, sean éstos del tipo que fueren, se debe a una decisión personal que realiza un empresario dado, hecho que no siempre se ajusta al mejor criterio empresarial.

Cuadro 6: Contribución de variables a cada grupo

\begin{tabular}{|l|r|r|r|r|r|r|r|c|}
\hline Variable & Gr. 1 & Gr. 2 & Gr. 3 & Gr. 4 & Gr. 5 & Gr. 6 & Gr. 7 & $\mathbf{R}^{\mathbf{2}}$ \\
\hline Altitud máxima & 0,072 & 0,356 & 0,492 & 0,000 & 0,264 & 0,085 & 0,576 & 0,607 \\
\hline Bien de Interés Cultural & 0,037 & 0,074 & 0,074 & 0,000 & 0,000 & 0,037 & 0,074 & 0,789 \\
\hline Museos y centros de interpretación & 0,100 & 0,200 & 0,300 & 0,000 & 0,000 & 0,000 & 0,000 & 0,770 \\
\hline Plazas en alojamientos hoteleros & 0,080 & 0,078 & 0,194 & 0,000 & 0,023 & 0,038 & 0,021 & 0,824 \\
\hline Plazas en alojamientos rurales & 0,184 & 0,454 & 0,546 & 0,000 & 0,480 & 0,158 & 0,237 & 0,405 \\
\hline Plazas en Restaurantes & 0,000 & 0,077 & 0,057 & 0,000 & 0,016 & 0,017 & 0,038 & 0,824 \\
\hline Población & 0,007 & 0,017 & 0,023 & 0,000 & 0,010 & 0,004 & 0,004 & 0,878 \\
\hline Superficie de embalses & 0,192 & 0,000 & 0,050 & 0,000 & 0,022 & 0,000 & 0,000 & 0,375 \\
\hline Superficie en parque Nacional & 0,205 & 0,000 & 0,000 & 0,000 & 0,000 & 0,000 & 0,000 & 0,878 \\
\hline Superficie en parque natural & 0,000 & 0,000 & 0,000 & 0,000 & 0,000 & 0,164 & 0,000 & 0,524 \\
\hline Zonas de baño/piscinas naturales & 0,000 & 0,143 & 0,429 & 0,000 & 0,429 & 0,000 & 0,143 & 0,701 \\
\hline Variable & $\mathbf{G r . 8}$ & $\mathbf{G r . 9}$ & $\mathbf{G r . ~ 1 0}$ & $\mathbf{G r . 1 1}$ & $\mathbf{G r . ~ 1 2}$ & $\mathbf{G r . ~ 1 3}$ & $\mathbf{G r . 1 4}$ & $\mathbf{R}^{\mathbf{2}}$ \\
\hline Altitud máxima & 0,253 & 0,550 & 0,179 & 0,283 & 0,068 & 0,000 & 0,763 & 0,607 \\
\hline Bien de Interés Cultural & 0,037 & 0,185 & 0,074 & 0,111 & 0,259 & 0,000 & 0,963 & 0,789 \\
\hline Museos y centros de interpretación & 0,100 & 0,100 & 0,000 & 0,200 & 0,500 & 0,000 & 0,600 & 0,770 \\
\hline Plazas en alojamientos hoteleros & 0,177 & 0,076 & 0,009 & 0,099 & 0,317 & 0,000 & 1,000 & 0,824 \\
\hline Plazas en alojamientos rurales & 0,188 & 0,342 & 0,201 & 0,520 & 0,319 & 0,000 & 0,757 & 0,405 \\
\hline Plazas en Restaurantes & 0,259 & 0,078 & 0,015 & 0,066 & 0,207 & 0,000 & 0,749 & 0,824 \\
\hline Población & 0,246 & 0,086 & 0,002 & 0,041 & 0,240 & 0,000 & 0,638 & 0,878 \\
\hline Superficie de embalses & 1,000 & 0,395 & 0,294 & 0,000 & 0,180 & 0,000 & 0,591 & 0,375 \\
\hline Superficie en parque Nacional & 0,000 & 0,000 & 0,352 & 0,000 & 0,138 & 0,000 & 0,000 & 0,878 \\
\hline Superficie en parque natural & 0,000 & 0,000 & 0,000 & 0,000 & 0,000 & 0,000 & 1,000 & 0,524 \\
\hline Zonas de baño/piscinas naturales & 0,143 & 0,429 & 0,000 & 0,000 & 0,143 & 0,000 & 0,143 & 0,701 \\
\hline
\end{tabular}

Fuente: Elaboración propia 
Sánchez Martín J. M., Rengifo Gallego J. I., Sánchez Rivero M. (2017): “Caracterización espacial del turismo en Extremadura mediante análisis de agrupamiento (Grouping Analysis). Un ensayo técnico”, GeoFocus (Artículos), $n^{\circ} 19$, p. 207-235. ISSN: 1578-5157. http://dx.doi.org/10.21138/GF.490

La caracterización e integración territorial de los grupos resultantes (véase cuadro 6 y figura 7) presenta como peculiaridad que algunos municipios, como Badajoz, Cáceres, Mérida o Trujillo, son los únicos representantes de sus grupos. Estos municipios se caracterizan por ser destinos consolidados del turismo cultural y/o urbano de Extremadura, aunque presentan lógicas diferencias entre ellos, ya sea por el volumen de población que reside en ellos, ya sea por los atractivos que poseen o por la propia oferta de alojamientos y restauración que presentan. A pesar de su relativa proximidad, cuando se observa la representación de sus términos municipales, no se puede pasar por alto su lejanía cuando se analizan las ciudades, entidad que se toma como referencia para hacer el análisis, pues la distancia entre Cáceres y Mérida supera los $60 \mathrm{~km}$. y entre Cáceres y Badajoz, supera los 90. Es decir, que no pueden formar parte del mismo grupo debido a la distancia tan elevada que presentan. No obstante, también se observa que existen diferencias entre los valores de los diferentes parámetros que los integran.

Las zonas de montaña del norte de Extremadura representan grupos variados y diferenciados, cada uno de ellos con peculiaridades significativas, pese a que tienen como criterios comunes la elevada altitud máxima y la presencia de zonas de baño, representadas, en este caso, por las piscinas naturales, a la vez que disponen de elevada oferta de alojamientos rurales. Además, conviene referir que también existen notables diferencias en cuanto a la riqueza cultural que atesoran, así como a la presencia de alojamientos hoteleros.

Así mismo, existe otra zona muy bien definida en el territorio, correspondiente a la comarca de La Serena y parte de las Vegas Altas del Guadiana, donde el principal factor condicionante es la presencia de grandes masas de agua embalsada. Sin embargo, dado que abarca a dos grandes núcleos de población, como Don Benito y Villanueva de la Serena, cabe destacar que también tienen cierta representatividad la oferta de alojamientos hoteleros y la restauración.

El municipio de Alcántara también se erige como grupo en el que destacan como variables significativas la presencia de un Parque Natural (Tajo Internacional), acompañado de un rico y variado patrimonio cultural, representado por numerosos BIC. Resulta destacable el hecho de que, a pesar de que en toda la zona rayana de la comarca de Alcántara el peso del Parque Natural es importante, casi ningún otro municipio atesora la calidad de los atractivos culturales del núcleo de Alcántara.

Sin lugar a dudas, el grupo 14, el más amplio desde el punto de vista territorial, y el más complejo, ya que no destaca en ninguna de las variables que se han considerado para el análisis. Esto no indica de ningún modo que carezcan de interés, pues hay núcleos, como Zafra, Plasencia, Coria y Olivenza, entre otros, que sí tienen cierto interés cultural, aunque su entorno más inmediato carece de él. Por consiguiente, resulta complicado formar un grupo homogéneo, a la vez que tampoco resultan tan determinantes como los grupos compuestos por un solo municipio.

La suma de los parámetros considerados en cada uno de los municipios refleja la realidad que presenta la distribución territorial de la actividad turística en Extremadura, permitiendo observar y comprender de forma fehaciente la diferenciación de cada territorio descrito, ya sea mediante el volumen de oferta, ya sea a través de los atractivos naturales y culturales o la propia población. De ese modo se observa que los grupos donde predominan los alojamientos rurales tienen como característica destacada la elevada altitud promedio de sus términos municipales, excepto los que configuran el grupo 14, que, como fue señalado anteriormente, es muy heterogéneo y permite sacar pocas conclusiones. También estos mismos grupos presentan marcadas diferencias entre ellos, 
Sánchez Martín J. M., Rengifo Gallego J. I., Sánchez Rivero M. (2017): “Caracterización espacial del turismo en Extremadura mediante análisis de agrupamiento (Grouping Analysis). Un ensayo técnico”, GeoFocus (Artículos), $n^{\circ} 19$, p. 207-235. ISSN: 1578-5157. http://dx.doi.org/10.21138/GF.490

como la mayor o menor presencia de equipamientos o recursos vinculados al turismo rural, como pueden ser la presencia de zonas de baño o atractivos culturales y otro tipo de oferta. Además, el volumen de población es relativamente reducido en buena parte de los casos.

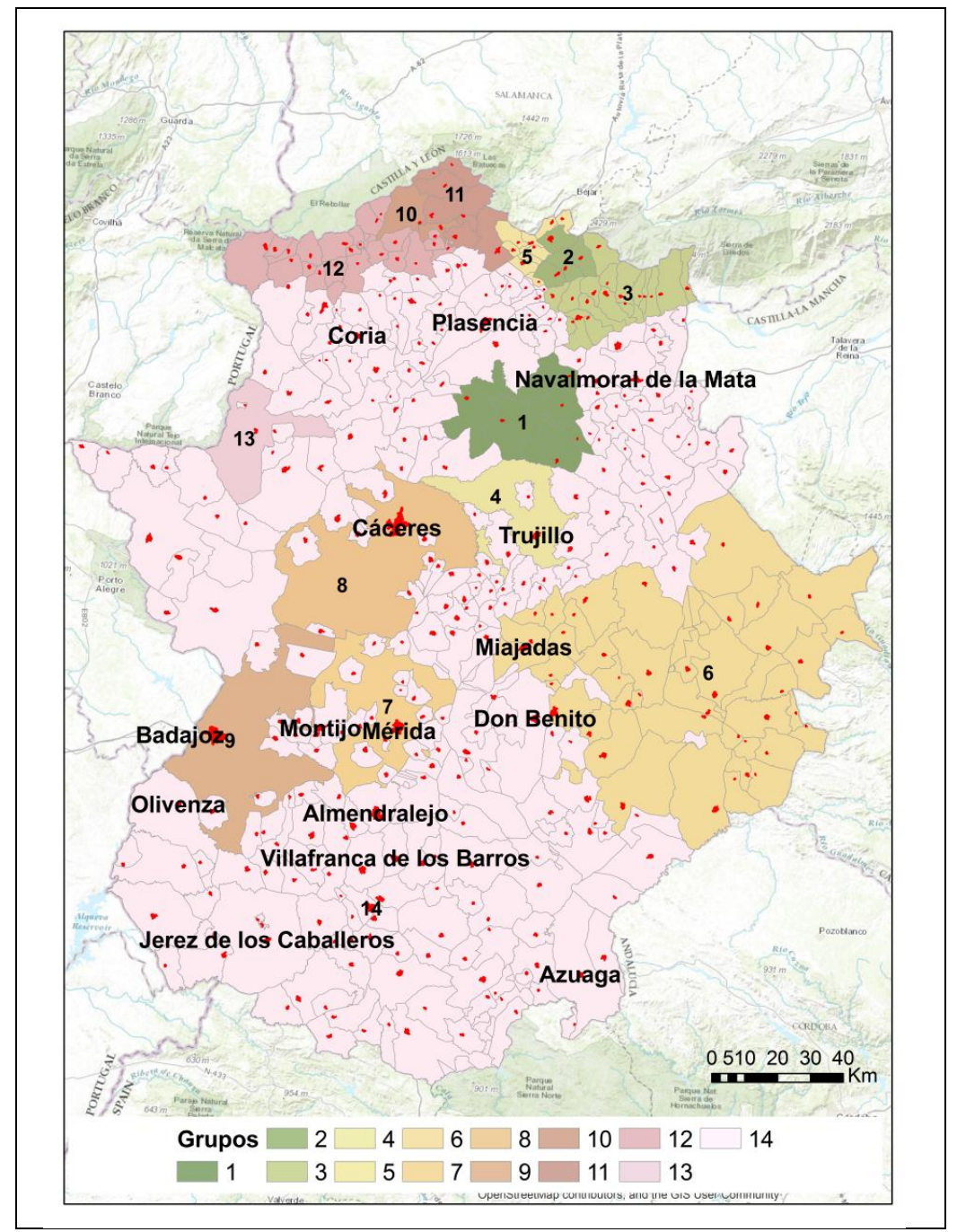

Figura 7: Análisis de grupos con número de vecinos (3)

Fuente: Elaboración propia

Como contraposición a los anteriores, muy concentrados en el norte de la provincia de Cáceres, aparecen los núcleos vinculados de forma directa al turismo cultural, formado por las principales ciudades y algún otro núcleo, como Trujillo, de reconocida fama como destino cultural. Estos grupos se caracterizan por disponer de elevada oferta hotelera y de restauración, a la vez que atesoran un rico patrimonio cultural y un volumen importante de población. A pesar de ello, también existen ciertas diferencias entre ellos, lo que da lugar a matices específicos lo suficientemente importantes como para que se pueda hablar de unidades diferenciadas. Otros grupos 
Sánchez Martín J. M., Rengifo Gallego J. I., Sánchez Rivero M. (2017): “Caracterización espacial del turismo en Extremadura mediante análisis de agrupamiento (Grouping Analysis). Un ensayo técnico”, GeoFocus (Artículos), $n^{\circ} 19$, p. 207-235. ISSN: 1578-5157. http://dx.doi.org/10.21138/GF.490

se confeccionan con atractivos naturales diferentes, como la presencia de parques naturales o nacionales y zonas con presencia de grandes embalses. En estos casos, quedan patentes sus características, apareciendo además muy concentrados en el territorio.

El análisis de las características de cada grupo (véase cuadro 7) refleja la complejidad del espacio turístico de Extremadura, pero muestra las capacidades que ofrece este tipo de análisis, al discriminar zonas muy conocidas dentro del contexto turístico.

Cuadro 7: Componentes de los grupos obtenidos

\begin{tabular}{|c|c|c|}
\hline Grupo & Características principales & $\begin{array}{l}\text { Municipios integrantes } \\
\text { (APÉNDICE 1) }\end{array}$ \\
\hline 1 & Superficie de Parque Nacional & $\begin{array}{l}\text { Entorno del parque } \\
\text { Nacional de Monfragüe }\end{array}$ \\
\hline 2 & $\begin{array}{l}\text { Altitud máxima, plazas en alojamientos rurales, zonas de } \\
\text { baño/piscinas naturales }\end{array}$ & Valle del Jerte \\
\hline 3 & $\begin{array}{l}\text { Altitud máxima, plazas en alojamientos rurales, museos y } \\
\text { centros de interpretación, zonas de baño/piscinas } \\
\text { naturales }\end{array}$ & La Vera \\
\hline 4 & $\begin{array}{l}\text { Plazas en alojamientos hoteleros, plazas en restaurantes, } \\
\text { bien de interés cultural, plazas en alojamientos rurales }\end{array}$ & Trujillo \\
\hline 5 & $\begin{array}{l}\text { Altitud máxima, zonas de baño/piscinas naturales, plazas } \\
\text { en alojamientos rurales }\end{array}$ & Valle del Ambroz \\
\hline 6 & Superficie de embalses & La Serena \\
\hline 7 & $\begin{array}{l}\text { Superficie en parque natural, museos y centros de } \\
\text { interpretación, bien de interés cultural, plazas en } \\
\text { alojamientos hoteleros, plazas en restaurantes, población }\end{array}$ & Mérida \\
\hline 8 & $\begin{array}{l}\text { Plazas en alojamientos hoteleros, plazas en restaurantes, } \\
\text { población, museos y centros de interpretación, bien de } \\
\text { interés cultural }\end{array}$ & Cáceres \\
\hline 9 & $\begin{array}{l}\text { Población, plazas en alojamientos hoteleros, plazas en } \\
\text { restaurantes }\end{array}$ & Badajoz \\
\hline 10 & $\begin{array}{l}\text { Zonas de baño/piscinas naturales, altitud máxima, plazas } \\
\text { en alojamientos rurales }\end{array}$ & Hurdes occidentales \\
\hline 11 & $\begin{array}{l}\text { Museos y centros de interpretación, altitud máxima, } \\
\text { superficie de embalses, plazas en alojamientos rurales }\end{array}$ & Hurdes orientales \\
\hline 12 & $\begin{array}{l}\text { Bienes de interés cultural, zonas de baño/piscinas } \\
\text { naturales, museos y centros de interpretación, altitud } \\
\text { máxima, plazas en alojamientos rurales }\end{array}$ & Sierra de Gata \\
\hline 13 & $\begin{array}{l}\text { Superficie de parque natural, museos y centros de } \\
\text { interpretación, superficie de embalses, bienes de interés } \\
\text { cultural }\end{array}$ & Alcántara \\
\hline 14 & $\begin{array}{l}\text { Aglutina casi todas las variables, sin destacar ninguna de } \\
\text { forma especial }\end{array}$ & Resto del territorio \\
\hline
\end{tabular}

Fuente: Elaboración propia 
Sánchez Martín J. M., Rengifo Gallego J. I., Sánchez Rivero M. (2017): “Caracterización espacial del turismo en Extremadura mediante análisis de agrupamiento (Grouping Analysis). Un ensayo técnico”, GeoFocus (Artículos), $n^{\circ} 19$, p. 207-235. ISSN: 1578-5157. http://dx.doi.org/10.21138/GF.490

\section{Discusión}

El sistema turístico de Extremadura es complejo, pues coexisten áreas donde el turismo está consolidado con otras donde su nivel de desarrollo es limitado o inexistente. En la mayor parte de los casos, el grado de progreso de la actividad depende de la vocación turística que le confieren los numerosos atractivos de los que goza el territorio, aunque no siempre es así, como se deduce de la complejidad para definir tipologías turísticas concretas en buena parte del territorio (Sánchez, 1998).

La mayor parte de las técnicas utilizadas para determinar y caracterizar el territorio turístico se ciñen a la aplicación de estadística multivariante y de carácter cuantitativo, para posteriormente cartografiar los resultados. Éstos posibilitan la concreción de tipologías turísticas definidas por los principales atributos del sistema. No obstante, cuando se plasman los resultados sobre el territorio, se observa que no siempre se configura un espacio turístico unificado y similar en sus características básicas. Por el contrario, a veces se muestra un espacio disperso y carente de unidad territorial (Sánchez et al, 2013; Sánchez et al, 2014), hecho que dificulta enormemente las tareas de planificación turística a escala plurimunicipal, especialmente si se pretende crear productos turísticos homogéneos y diferenciados.

Debido a esas circunstancias, se ha considerado oportuno realizar un ensayo técnico de aplicación de Análisis de Agrupamiento (Grouping Analysis) para la caracterización espacial del turismo en Extremadura. Esta técnica, además de tomar como referencia los atributos numéricos, tiene en cuenta la componente espacial. Esta componente resulta clave cuando se trata es de dar consistencia territorial a la caracterización del turismo, de cara a la mejora de su gestión. Así, la consideración de esta componente permite la configuración de unidades territoriales próximas en el espacio y que, además, disponen de características comunes. Con ello se puede fortalecer el sistema turístico en escalas intermedias y plurimunicipales, permitiendo la creación y explotación de productos turísticos conjuntos, apoyados por la propia contigüidad espacial.

La aplicación de esta técnica ofrece múltiples configuraciones para la definición del criterio espacial. Los ajustes espaciales dependen del tipo de geometría utilizada, así como de su contigüidad en el territorio. En el caso que nos ocupa, se han utilizado los núcleos de población (capitales municipales). Al ser elementos dispersos, las posibilidades se reducen a la triangulación de Delaunay y al número de vecinos próximos. No obstante, también se puede otorgar un peso espacial partiendo de otras variables no consideradas. A su vez, también está presente la opción de no incluir ningún criterio espacial. Si se utiliza como geometría de análisis el término municipal, con continuidad en el territorio, las posibilidades de elección de criterio espacial se incrementan, aunque los resultados se desvirtúan, dada la configuración de cada entidad territorial, pues, en el caso que nos ocupa, coexisten municipios de más de $1.750 \mathrm{~km}^{2}$ con otros que apenas alcanzan 3,7 $\mathrm{km}^{2}$.

Los resultados que se obtienen de este trabajo permiten inferir que la utilización de la estadística espacial, representada en este caso por el análisis de agrupamiento, utilizando restricciones espaciales a través del criterio de vecindad, tiene ventajas sobre técnicas como el análisis factorial, más potente en el análisis de variables, pero carente de relaciones espaciales. 
Sánchez Martín J. M., Rengifo Gallego J. I., Sánchez Rivero M. (2017): “Caracterización espacial del turismo en Extremadura mediante análisis de agrupamiento (Grouping Analysis). Un ensayo técnico”, GeoFocus (Artículos), $n^{\circ} 19$, p. 207-235. ISSN: 1578-5157. http://dx.doi.org/10.21138/GF.490

A pesar de ello, trabajar con este tipo de herramienta geoestadística no está exento de limitaciones, que vienen dadas no solo por la necesidad de simplificar y reducir el número de variables, sino también por la disposición de elementos territoriales que se analicen. Así, si éstos no presentan una distribución relativamente homogénea en el territorio, es posible que aparezcan grupos diferenciados debido a la gran distancia que los separa. Esto cobra especial relevancia en ámbitos como el analizado, donde los principales exponentes del turismo cultural, por ejemplo, presenta una gran distancia entre sus centros, pese a que se trata de municipios colindantes. Obviamente, este tipo de relación podría haberse modificado, utilizando como unidad de análisis el término municipal y no el núcleo de población. Sin embargo, desde el punto de vista metodológico no parece conveniente aplicar este criterio, dado que la oferta de alojamientos se concentra precisamente en los núcleos de población. En definitiva, la aplicación de esta técnica exige la realización de numerosas pruebas, con el fin de adaptar la restricción espacial, dependiendo de la zona y de las unidades territoriales analizadas. Por otro lado, cuando se aplica un criterio de distancia, ésta necesariamente debe ser euclidiana o Manhattan, no permitiendo utilizar la distancia real medida a través de las vías de comunicación. Por ello, cabría plantear, como perspectiva de trabajo, la necesidad de implementar en esta técnica el análisis de redes como criterio básico para establecer el concepto de distancia real.

\section{Conclusiones}

Tras la realización de este ensayo técnico extraemos las siguientes conclusiones:

En el caso de Extremadura, la presencia de determinados atractivos, la oferta de alojamientos y de restauración, así como el volumen demográfico son los factores principales que permiten caracterizar la mayor parte del espacio turístico a partir de la aplicación de técnicas de análisis de agrupamiento (Grouping Analysis).

La unidad territorial de análisis más adecuada para el desarrollo de esta aplicación es la conformada por las capitales de los términos municipales, ya que, si se considera el territorio completo, existen ciertos problemas metodológicos. Para mayor simplicidad en los cálculos, tanto de distancia euclidiana, como de vecino próximo, resulta más adecuado utilizar el centroide interno de los núcleos de población.

Existe una diferencia notoria entre la aplicación de la restricción espacial y su omisión. La inclusión de la componente territorial parece más adecuada cuando se busca obtener zonas homogéneas no solo en su composición, sino también en su configuración territorial. La incorporación de una restricción espacial permite definir y caracterizar más grupos, que muestran una mayor consistencia como territorios unificados.

La restricción espacial más útil y acertada, tanto desde el punto de vista metodológico, es la que considera los vecinos próximos, frente a la triangulación de Delaunay.

De la aplicación de las técnicas de análisis de agrupamiento se obtiene, en el caso de Extremadura, una zona de incertidumbre, de indefinición, muy extensa, fiel reflejo de la realidad que muestra el territorio turístico de esta Comunidad. Se constata la distribución del sistema 
Sánchez Martín J. M., Rengifo Gallego J. I., Sánchez Rivero M. (2017): “Caracterización espacial del turismo en Extremadura mediante análisis de agrupamiento (Grouping Analysis). Un ensayo técnico”, GeoFocus (Artículos), $n^{\circ} 19$, p. 207-235. ISSN: 1578-5157. http://dx.doi.org/10.21138/GF.490

turístico rural en las zonas que tienen los atractivos preferidos por la demanda, configurando una diversidad de espacios turísticos en el norte de la provincia de Cáceres. Al mismo tiempo, aparecen otros espacios turísticos, muy bien delimitados, como los que presentan una clara vinculación al potencial que tienen los grandes embalses y los espacios naturales para la práctica del turismo.

Finalmente, se concluye que el análisis de agrupamiento es una técnica consistente e interesante para caracterizar territorios turísticos homogéneos y que presenten cierta continuidad espacial. No obstante, también presenta ciertas limitaciones, como la necesidad de realizar numerosas pruebas de adaptación, o la estanqueidad a la hora de aplicar criterios de distancia.

\section{Referencias bibliográficas}

Antón Clavé, S. y González Reverte, F. (2011): Planificación territorial del turismo. Barcelona, UOC.

Boers, B. y Cottrell, S. (2007): "Sustainable Tourism Infrastructure Planning: A GIS-Supported Approach", Tourism Geographies: An International Journal of Tourism Space, Place and Environment, pp. 1-21.

Calinski, T. y Harabasz, J. (1974): "A dendrite method for cluster analysis", Communications in Statistics, 3 (1), pp. 1-27.

Chancellor, C. Y. (2008): "Using Geographic Information System to Visualize Travel Patterns and Market Research Data", Journal of Travel y Tourism Marketing, 25(3-4), pp. 341-354.

Fang, Y., Huang, Z., Wang, K. y Cai, B. (2015): "Spatial Pattern of Chinese Tourism Development and Its Mechanism Based on Different Spatial-temporal Scales: Taking the Panel Data of China Mainland (1996-2010) for Example", Journal of Landscape Research, 7 (2), pp. 47-54.

García-Palomares, J., Gutiérrez, J., y Mínguez, C. (2015): "Identification of tourist hot spots based on social networks: A comparative analysis of European metropolises using photo-sharing services and GIS", Applied Geography, 63, pp. 408-417.

Gunn, C. (1988): Tourism planning. London, Taylor y Francis.

Haggett, P. (1976): Análisis locacional en la geografía humana. Barcelona: Gustavo Gili.

INE (varios años): Instituto Nacional de Estadística. [Consulta: 18-7-2015]. Disponible en ww.ine.es

JUNTAEX, D. G. de Turismo (2010): turismoextremadura.com. [Consulta: 18-12-2015].

Disponible en turismoextremadura.com

Rengifo Gallego, J.I., Sánchez Martín, J.M., y Sánchez Rivero, M. (2013): “Análisis del desarrollo del turismo rural en la provincia de Cáceres en los inicios del S.XXI", Pasos. Revista de Turismo y Patrimonio Cultural, 11 (4), pp. 615-630.

Ritchie, B., Burns, P., y Palmer, C. (2005): Tourism Research Methods: Integrating Theory with Practice. Oxfordshire, CABI.

Sánchez Martín, J. M. (1998): “Tipología turística municipal de Extremadura basada en el Análisis Factorial de Componentes Principales”, Lurralde. Investigación y espacio, 21, pp. 95-119. 
Sánchez Martín J. M., Rengifo Gallego J. I., Sánchez Rivero M. (2017): “Caracterización espacial del turismo en Extremadura mediante análisis de agrupamiento (Grouping Analysis). Un ensayo técnico”, GeoFocus (Artículos), $n^{\circ} 19$, p. 207-235. ISSN: 1578-5157. http://dx.doi.org/10.21138/GF.490

Sánchez Martín, J.M., Sánchez Rivero, M., y Rengifo Gallego, J. (2013): "La evaluación del potencial para el desarrollo del turismo rural. Aplicación metodológica sobre la provincia de Cáceres", Geofocus. Revista Internacional de Ciencia y Tecnología de la Información Geográfica, 13 (1), pp. 99-130.

Sánchez Martín, J.M., Sánchez Rivero, M., y Rengifo Gallego, J. (2017): “Análisis del equilibrio entre el potencial turístico y la oferta de alojamientos en turismo rural mediante técnicas de estadística espacial. Una aplicción a la provincia de Cáceres (España)", Cuadernos de Turismo, 39, pp. 547-576.

Sánchez Rivero, M. (2008): "Análisis espacial de datos y turismo: nuevas técnicas para el análisis turístico. Una aplicación al caso extremeño", Revista de Estudios Empresariales. Segunda época, 2, pp. 48-66.

Sánchez Rivero, M., Sánchez Martín, J.M., y Rengifo Gallego, J. (2014): "Methodological approach for assessing the potential of a rural tourism destination: An application in the province of Cáceres (Spain)”, Current Issues in Tourism, pp. 1-19.

Tobler, W. A. (1970): "Computer Movie Simulating Urban Growth in the Detroit Region", Economic Geography, 46, pp. 234-240.

Yokeno, N. (1968): "La localisation de l'industrie touristique: application de l'analyse de ThunenWeber", Cahiers de tourisme 9, pp. 163-188.

\section{APÉNDICE 1: Municipios integrantes de cada grupo}

Grupo 1: Casas de Miravete, Jaraicejo, Serradilla, Serrejón, Toril y Torrejón el Rubio.

Grupo 2: Cabezuela del Valle, Hervás, Jerte y Navaconcejo.

Grupo 3: Aldeanueva de la Vera, Collado de la Vera, Cuacos de Yuste, Garganta la Olla, Guijo de Santa Bárbara, Jaraíz de la Vera, Jarandilla de la Vera, Losar de la Vera, Madrigal de la Vera, Piornal, Robledillo de la Vera, Talaveruela de la Vera, Tornavacas, Torremenga, Valverde de la Vera, Viandar de la Vera y Villanueva de la Vera.

Grupo 4: Trujillo.

Grupo 5: Abadía, Aldeanueva del Camino, Baños de Montemayor, Casas del Monte, Garganta (La), Gargantilla, Granja (La), Rebollar y Segura de Toro.

Grupo 6: Abertura, Acedera, Alcollarín, Alía, Baterno, Cabeza del Buey, Campanario, Campo Lugar, Capilla, Casas de Don Pedro, Castilblanco, Castuera, Coronada (La), Escurial, Esparragosa de Lares, Fuenlabrada de los Montes, Garbayuela, Garlitos, Helechosa de los Montes, Herrera del Duque, Logrosán, Madrigalejo, Miajadas, Navalvillar de Pela, Orellana de la Sierra, Orellana la Vieja, Peñalsordo, Puebla de Alcocer, Rena, Risco, Sancti-Spíritus, Siruela, Talarrubias, Tamurejo, Valdecaballeros, Villamesías, Villanueva de la Serena, Villar de Rena, Villarta de los Montes, Zarza-Capilla y Zorita.

Grupo 7: Mérida.

Grupo 8: Cáceres. 
Sánchez Martín J. M., Rengifo Gallego J. I., Sánchez Rivero M. (2017): “Caracterización espacial del turismo en Extremadura mediante análisis de agrupamiento (Grouping Analysis). Un ensayo técnico”, GeoFocus (Artículos), $n^{\circ} 19$, p. 207-235. ISSN: 1578-5157. http://dx.doi.org/10.21138/GF.490

Grupo 9: Badajoz.

Grupo 10: Casar de Palomero y Pinofranqueado.

Grupo 11: Caminomorisco, Casares de Hurdes, Ladrillar, Mohedas de Granadilla, Nuñomoral , Pesga (La) y Zarza de Granadilla.

Grupo 12: Acebo, Cadalso, Cerezo, Descargamaría, Eljas, Gata, Hernán-Pérez, Hoyos, Marchagaz, Palomero, Perales del Puerto, Robledillo de Gata, San Martín de Trevejo, Santa Cruz de Paniagua, Santibáñez el Alto, Torre de Don Miguel, Torrecilla de los Ángeles, Valverde del Fresno, Villamiel, Villanueva de la Sierra y Villasbuenas de Gata.

Grupo 13: Alcántara

Grupo 14: Acehúche, Aceituna, Aceuchal, Ahigal, Ahillones, Alagón del Río, Alange, Albalá, Albuera (La), Alburquerque, Alconchel, Alconera, Alcuéscar, Aldea del Cano, Aldea del Obispo (La), Aldeacentenera, Aldehuela de Jerte, Aliseda, Aljucén, Almaraz, Almendral, Almendralejo, Almoharín, Arroyo de la Luz, Arroyo de San Serván, Arroyomolinos, Arroyomolinos de la Vera, Atalaya, Azuaga, Barcarrota, Barrado, Belvís de Monroy, Benquerencia, Benquerencia de la Serena, Berlanga, Berrocalejo, Berzocana, Bienvenida, Bodonal de la Sierra, Bohonal de Ibor, Botija, Brozas, Burguillos del Cerro, Cabañas del Castillo, Cabeza la Vaca, Cabezabellosa, Cabrero, Cachorrilla, Calamonte, Calera de León, Calzadilla, Calzadilla de los Barros, Campillo de Deleitosa, Campillo de Llerena, Cañamero, Cañaveral, Carbajo, Carcaboso, Carmonita, Carrascalejo, Carrascalejo (El), Casar de Cáceres, Casas de Don Antonio, Casas de Don Gómez, Casas de Millán, Casas de Reina, Casas del Castañar, Casatejada, Casillas de Coria, Castañar de Ibor, Ceclavín, Cedillo, Cheles, Cilleros, Codosera (La), Conquista de la Sierra, Cordobilla de Lácara, Coria, Corte de Peleas, Cristina, Cumbre (La), Deleitosa, Don Benito, Don Álvaro, Entrín Bajo, Esparragalejo, Esparragosa de la Serena, Feria, Fregenal de la Sierra, Fresnedoso de Ibor, Fuente de Cantos, Fuente del Arco, Fuente del Maestre, Fuentes de León, Galisteo, Garciaz, Gargüera, Garrovilla (La), Garrovillas de Alconétar, Garvín, Gordo (El), Granja de Torrehermosa, Guadalupe, Guadiana del Caudillo, Guareña, Guijo de Coria, Guijo de Galisteo, Guijo de Granadilla, Haba (La), Herguijuela, Herrera de Alcántara, Herreruela, Higuera, Higuera de la Serena, Higuera de Llerena, Higuera de Vargas, Higuera la Real, Hinojal, Hinojosa del Valle, Holguera, Hornachos, Huélaga, Ibahernando, Jarilla, Jerez de los Caballeros, Lapa (La), Llera, Llerena, Lobón, Madroñera, Magacela, Maguilla, Majadas, Malcocinado, Malpartida de Cáceres, Malpartida de la Serena, Malpartida de Plasencia, Manchita, Mata de Alcántara, Medellín, Medina de las Torres, Membrío, Mengabril, Mesas de Ibor, Millanes, Mirabel, Mirandilla, Monesterio, Monroy, Montánchez, Montehermoso, Montemolín, Monterrubio de la Serena, Montijo, Moraleja, Morcillo, Morera (La), Nava de Santiago (La), Navalmoral de la Mata, Navalvillar de Ibor, Navas del Madroño, Navezuelas, Nogales, Oliva de la Frontera, Oliva de Mérida, Oliva de Plasencia, Olivenza, Palomas, Parra (La), Pasarón de la Vera, Pedroso de Acim, Peraleda de la Mata, Peraleda de San Román, Peraleda del Zaucejo, Pescueza, Piedras Albas, Plasencia, Plasenzuela, Portaje, Portezuelo, Pozuelo de Zarzón, Puebla de la Calzada, Puebla de la Reina, Puebla de Obando, Puebla de Sancho Pérez, Puebla del Maestre, Puebla del Prior, Pueblonuevo de Miramontes, Pueblonuevo del Guadiana, Puerto de Santa Cruz, Quintana de la Serena, Reina, Retamal de Llerena, Ribera del Fresno, Riolobos, Robledillo de Trujillo, Robledollano, Roca de la Sierra (La), Romangordo, Rosalejo, Ruanes, Salorino, Salvaleón, Salvatierra de los Barros, Salvatierra de Santiago, San Pedro de Mérida, San Vicente de Alcántara, Santa Amalia, Santa Ana, Santa Cruz de la Sierra, Santa Marta, Santa Marta de Magasca, Santiago de Alcántara, Santiago del Campo, Santibáñez el Bajo, 
Sánchez Martín J. M., Rengifo Gallego J. I., Sánchez Rivero M. (2017): "Caracterización espacial del turismo en Extremadura mediante análisis de agrupamiento (Grouping Analysis). Un ensayo técnico”, GeoFocus (Artículos), $n^{\circ} 19$, p. 207-235. ISSN: 1578-5157. http://dx.doi.org/10.21138/GF.490

Santos de Maimona (Los), Saucedilla, Segura de León, Sierra de Fuentes, Solana de los Barros, Talaván, Talavera la Real, Talayuela, Táliga, Tejeda de Tiétar, Tiétar, Torno (El), Torre de Miguel Sesmero, Torre de Santa María, Torrecillas de la Tiesa, Torrejoncillo, Torremayor, Torremejía, Torremocha, Torreorgaz, Torrequemada, Trasierra, Trujillanos, Usagre, Valdastillas, Valdecañas de Tajo, Valdefuentes, Valdehúncar, Valdelacalzada, Valdelacasa de Tajo, Valdemorales, Valdeobispo, Valdetorres, Valencia de Alcántara, Valencia de las Torres, Valencia del Mombuey, Valencia del Ventoso, Valle de la Serena, Valle de Matamoros, Valle de Santa Ana, Valverde de Burguillos, Valverde de Leganés, Valverde de Llerena, Valverde de Mérida, Vegaviana, Villa del Campo, Villa del Rey, Villafranca de los Barros, Villagarcía de la Torre, Villagonzalo, Villalba de los Barros, Villanueva del Fresno, Villar de Plasencia, Villar del Pedroso, Villar del Rey, Zafra, Zahínos, Zalamea de la Serena, Zarza de Montánchez y Zarza la Mayor y Zarza (La). 